\title{
Khovanov homology for signed divides
}

\author{
Olivier Couture
}

The purpose of this paper is to interpret polynomial invariants of strongly invertible links in terms of Khovanov homology theory. To a divide, that is a proper generic immersion of a finite number of copies of the unit interval and circles in a 2-disc, one can associate a strongly invertible link in the 3 -sphere. This can be generalized to signed divides: divides with + or - sign assignment to each crossing point. Conversely, to any link $L$ that is strongly invertible for an involution $j$, one can associate a signed divide. Two strongly invertible links that are isotopic through an isotopy respecting the involution are called strongly equivalent. Such isotopies give rise to moves on divides. In a previous paper [2], the author finds an exhaustive list of moves that preserves strong equivalence, together with a polynomial invariant for these moves, giving therefore an invariant for strong equivalence of the associated strongly invertible links. We prove in this paper that this polynomial can be seen as the graded Euler characteristic of a graded complex of $\mathbb{Z}_{2}-$ vector spaces. Homology of such complexes is invariant for the moves on divides and so is invariant through strong equivalence of strongly invertible links.

$57 \mathrm{M} 27$

\section{Introduction}

A divide $\Gamma$ is the image of a proper generic immersion of a finite number of intervals and circles into the unit 2-disc $\mathbf{D}^{2}$ of $\mathbb{R}^{2}$. To a divide $\Gamma$, N A'Campo [1] associates a link $\mathcal{L}(\Gamma)$ in the unit 3 -sphere of the tangent space $T \mathbf{D}^{2}$ :

$$
\mathcal{L}(\Gamma)=\left\{(p, v) \in T \mathbf{D}^{2}: p \in \Gamma, v \in T_{p} \Gamma,\|p\|^{2}+\|v\|^{2}=1\right\} .
$$

This link has natural orientation and is strongly invertible with respect to the involution $j(p, v)=(p,-v)$.

Couture and Perron [3] give a generalization of divides. Let $(x, y)$ be coordinates in $\mathbf{D}^{2}$ such that the restriction to $\Gamma$ of the projection $\pi_{1}:(x, y) \mapsto x$ is a Morse function. A Morse signed divide (MS-divide) relative to $\pi_{1}$ stands for such a divide with + or - sign assignment to each double point of $\Gamma$. Furthermore, if there exists $a \in] 0,1[$ such that all maxima (resp. minima) of $\pi_{1 \mid \Gamma}$ project on $a$ (resp. $-a$ ) and all double points in $]-a, a[$, the MS-divide is called ordered (OMS-divide). 
We also define a link associated with a MS-divide (see Couture and Perron [3]), which is strongly invertible with respect to the involution $j(p, v)=(p,-v)$. If all signs are positive, this link is no more than the link of the divide without signs. The interest of OMS-divides rather than MS-divides is to obtain an immediate braid presentation of the link from the divide.

Strongly invertible links are closely related to OMS-divides. Let $L$ be an oriented link in $\mathbb{S}^{3}$ and $j$ be an involution of $\left(\mathbb{S}^{3}, L\right)$ with nonempty fixed point set, which preserves the orientation of $\mathbb{S}^{3}$ and reverses the orientation of $L$. Then $(L, j)$ is called a strongly invertible link. As we said above, the link of a divide is strongly invertible for the implicit strong inversion $j(p, v)=(p,-v)$. Two strongly invertible links $(L, j)$ and $\left(L^{\prime}, j^{\prime}\right)$ are strongly equivalent if there exists an isotopy $\varphi_{t}, t \in[0,1]$ of $\mathbb{S}^{3}$ sending $L$ to $L^{\prime}$ such that $\varphi_{1} \circ j=j^{\prime} \circ \varphi_{1}$.

Isotopies through MS-divides give rise to strong equivalence of associated links. Also, one can find in Couture [2] an (exhaustive) list of elementary moves on MS-divides that preserve strong equivalence classes of the associated links. As a particular case, given a MS-divide, we can always construct another one using these moves which is an OMS-divide. Besides, we can transpose the moves on MS-divides directly to moves on OMS-divides. Two OMS-divides obtained one from the other by those moves on OMS-divides are called $\mathcal{M}$-equivalent (see the list of moves in Section 1.3). Then as an essential result of [2], we have:

Theorem (Couture [2]) (1) Every strongly invertible link is strongly equivalent to the link of an OMS-divide.

(2) The links of two OMS-divides are strongly equivalent if and only if the OMSdivides are $\mathcal{M}$-equivalent.

As the Jones polynomial is invariant under Reidemeister moves on links diagrams, there exists a Laurent polynomial for an OMS-divide with integral coefficients (see Couture [2]), which is invariant under $\mathcal{M}$-equivalence and so invariant under strong equivalence of strongly invertible links. Modulo 2, this polynomial coincides with the Jones polynomial of the link. The purpose of this paper is to interpret the polynomial of an OMS-divide as the graded Euler characteristics of a graded complex of $\mathbb{Z}_{2}-$ vector spaces (Proposition 3.16). Besides, if we call the Khovanov homology of an OMS-divide the homology of this complex, then we have a stronger result:

Theorem 3.17 Khovanov homology of OMS-divides is invariant under $\mathcal{M}$-equivalence.

Corollary 3.18 Khovanov homology is an invariant for strong equivalence of strongly invertible links. 
Eventually, Khovanov homology of OMS-divides is a refinement of the polynomial invariant of OMS-divides.

This paper is dedicated to Bernard Perron.

\section{Divides and OMS-divides}

\subsection{Divides and links of divides}

A divide of the unit 2 -disc $\mathbf{D}^{2}$ of $\mathbb{R}^{2}(\simeq \mathbb{C})$ is the image $\Gamma$ of a proper generic immersion $\gamma$ :

$$
\gamma:(J, \partial J) \rightarrow\left(\mathbf{D}^{2}, \partial \mathbf{D}^{2}\right), \quad J=\left(\bigsqcup_{j=1}^{r} I_{j}\right) \sqcup\left(\bigsqcup_{j=1}^{s} S_{j}\right),
$$

where $I_{j}$ and $S_{j}$ are respectively copies of $[0,1]$ and $\mathbb{S}^{1}=\{z \in \mathbb{C}:|z|=1\}$ and generic means that the only singularities of $\gamma$ are ordinary double points and $\Gamma=$ $\gamma(J)$ intersects $\partial \mathbf{D}^{2}$ transversally. Every $\gamma\left(I_{j}\right)$ (resp. $\gamma\left(S_{j}\right)$ ) is called interval (resp. circular) branch.

Let $S\left(\mathbf{D}^{2}\right)=\left\{(p, v) \in T \mathbf{D}^{2}:|p|^{2}+|v|^{2}=1\right\}$ be the unit sphere of the tangent space $T \mathbf{D}^{2} \simeq \mathbf{D}^{2} \times \mathbb{C}$. To a divide, A'Campo [1] associates a link $\mathcal{L}(\Gamma)$ in $S\left(\mathbf{D}^{2}\right)$ :

$$
\mathcal{L}(\Gamma)=\left\{(p, v) \in S\left(\mathbf{D}^{2}\right): p \in \Gamma, v \in T_{p} \Gamma\right\} .
$$

This link has a natural orientation induced by the two possible orientations of the branches of $\Gamma$ and is strongly invertible for the involution $j(p, v)=(p,-v)$ of $S\left(\mathbf{D}^{2}\right)$ with axis $\operatorname{Fix}(j)=\partial \mathbf{D}^{2} \times\{0\}$ (see Section 1.3 below). Each interval branch of $\Gamma$ leads to a strongly invertible component of $\mathcal{L}(\Gamma)$ and each circular branch of $\Gamma$ to two components of $\mathcal{L}(\Gamma)$ interchanged by $j$.

\subsection{OMS-divides}

Let $\Gamma$ be a divide. Let $\rho(x+i y)=x$ be the projection on real axis. Suppose there exists $(a, b) \in] 0,1[\times] 0,1\left[, a^{2}+b^{2}<1\right.$ such that

(1) $\Gamma \subset\left\{x+i y \in \mathbf{D}^{2}:-b<y<b\right\}$ and the restriction $\rho_{\mid \Gamma}$ is a Morse function;

(2) all double points of $\Gamma$ are contained in $]-a, a[\times]-b, b[$;

(3) all maxima (resp. minima) of $\rho_{\mid \Gamma}$, called vertical tangent points, project onto $a$ (resp. $-a$ ). 
Then $\Gamma$ is called an ordered Morse divide. Double points and vertical tangent points will be called singular points of $\Gamma$. Now let $\epsilon$ be a function that associates a + or sign with each double point of $\Gamma$. Then $(\Gamma, \epsilon)$ is called an ordered Morse signed divide (OMS-divide) relative to the projection $\rho(x+i y)=x$ (see Couture and Perron [3]).

Let's associate an oriented $j$-strongly invertible link $\mathcal{L}(\Gamma, \epsilon)$ with an OMS-divide $(\Gamma, \epsilon)$. This link coincides with $\mathcal{L}(\Gamma)$ except in solid tori $T D_{p} \cap S\left(\mathbf{D}^{2}\right) \simeq D_{p} \times \mathbb{S}^{1}$ over small discs $D_{p}$ around negative double points $p$ of $(\Gamma, \epsilon)$ where we change the two $j$-symmetric crossings from over to under. More precisely, in such a solid torus $T D_{p} \cap S\left(\mathbf{D}^{2}\right)$, the link is defined according to Figure 1. If $\epsilon=+$ for all double points then $\mathcal{L}(\Gamma, \epsilon)=\mathcal{L}(\Gamma)$.
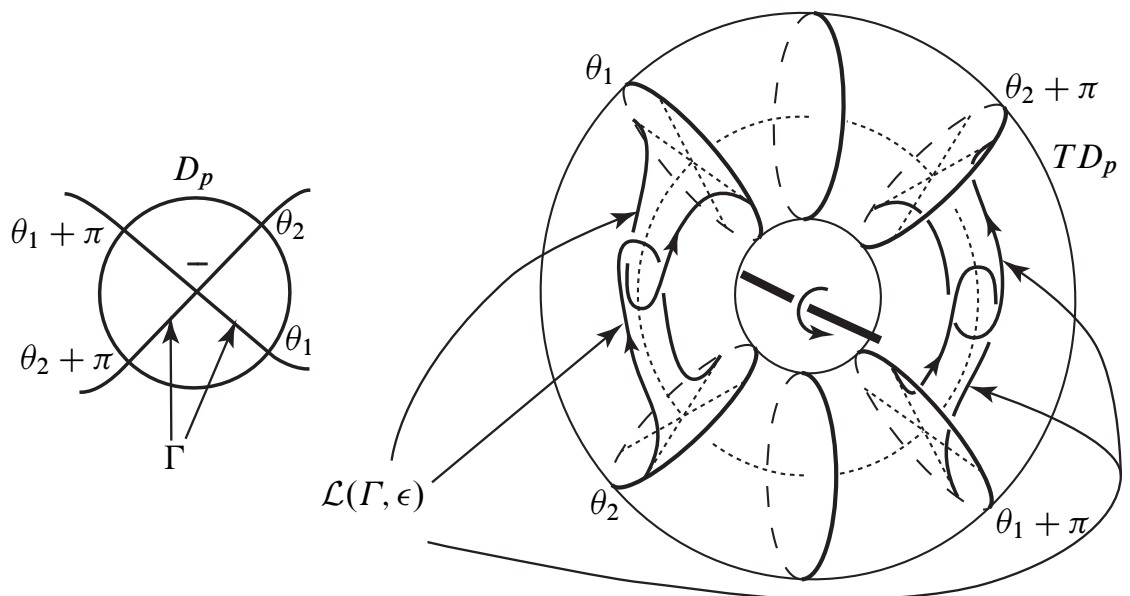

Figure 1: The link $\mathcal{L}(\Gamma, \epsilon)$ over a negative double point

Besides, from a divide $\Gamma$ one can construct an OMS-divide $\left(\Gamma^{\prime}, \epsilon\right)$ by a succession of moves and isotopies, such that $\mathcal{L}\left(\Gamma^{\prime}, \epsilon\right)$ and $\mathcal{L}(\Gamma)$ are isotopic [3] by an isotopy that respects the involution $j$ (see Section 1.3 below for a more precise definition).

Remark 1.1 For simplicity, we will only consider an OMS-divide $(\Gamma, \epsilon)$ in $[-a, a] \times$ $[-b, b]$, omitting its trivial part outside this rectangle. After rescaling, we also suppose that $a=b=1$. Since we will often consider diagrams of local parts of OMS-divides $(\Gamma, \epsilon)$, we distinguish end points of $\Gamma$, ie points of $\Gamma$ in $\{-1,1\} \times[-1,1]$ without vertical tangent by a big point (see Figure 2).

Moreover, we will simply write $\Gamma$ instead of $(\Gamma, \epsilon)$ if no ambiguity occurs in the context. 


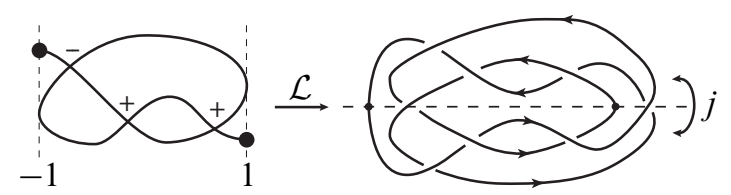

Figure 2: A representative OMS-divide for the strongly invertible knot $5_{2}$

\section{$1.3 \mathcal{M}$-Equivalence for OMS-divides}

Two OMS-divides $\Gamma$ and $\Gamma^{\prime}$ are $\mathcal{M}$-equivalent if we obtain one from the other by isotopy through OMS-divides and a finite sequence of the moves described in Figure 3 or symmetric situations with respect to horizontal and vertical directions (see Couture [2]). Let $j$ be an orientation preserving involution of $\mathbb{S}^{3}$ with nonempty fix
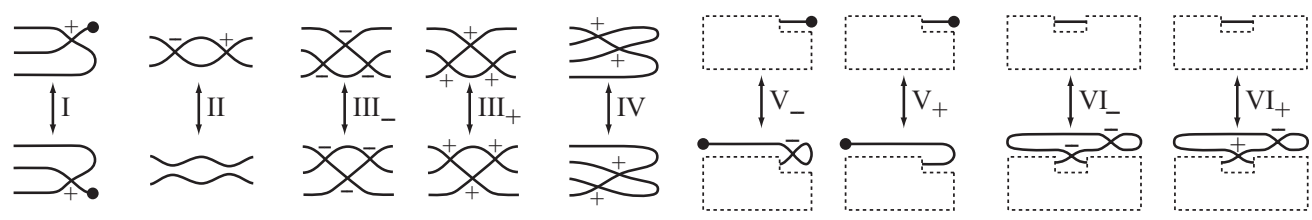

Figure 3: Moves of $\mathcal{M}$-equivalence

point set (ie $\operatorname{Fix}(j)$ is a trivial knot according to the solution of Smith conjecture; see Morgan and Bass [4]). An oriented link $L \subset \mathbb{S}^{3}$ is $j$-strongly invertible if $j$ sends $L$ to itself with opposite orientation. The couple $(L, j)$ is called a strongly invertible link. With the link of an OMS-divide, we implicitly associate natural orientation and involution $j(p, v)=(p,-v)$ as in Section 1.1: such a link is strongly invertible.

Two strongly invertible links $(L, j)$ and $\left(L^{\prime}, j^{\prime}\right)$ are called strongly equivalent ${ }^{1}$ if there exists and isotopy $\varphi_{t}, t \in[0,1]$ of $\mathbb{S}^{3}$ sending $L$ to $L^{\prime}$ such that $\varphi_{1} \circ j=j^{\prime} \circ \varphi_{1}$. One can easily prove that $\mathcal{M}$-equivalent OMS-divides give rise to strongly equivalent strongly invertible links. Conversely, let's recall the following crucial theorem relating OMS-divides with strongly invertible links.

Theorem 1.2 (Couture [2]) (1) Every strongly invertible link is strongly equivalent to the link of an OMS-divide.

(2) The links of two OMS-divides are strongly equivalent if and only if the OMSdivides are $\mathcal{M}$-equivalent.

\footnotetext{
${ }^{1}$ The same link $L$ may have two strong inversions $j$ and $j^{\prime}$ such that $(L, j)$ and $\left(L, j^{\prime}\right)$ are not strongly equivalent.
} 


\section{The polynomial of an OMS-divide}

Let's denote by $\Theta_{0}$ and $\Theta_{1}$ the local splittings of an OMS-divide $(\Gamma, \epsilon)$ in a neighborhood of a double point or vertical tangent point described in Figure $4\left(\Theta_{0}\right.$ "smoothes" the OMS-divide whereas $\Theta_{1}$ introduces horizontal cusps).
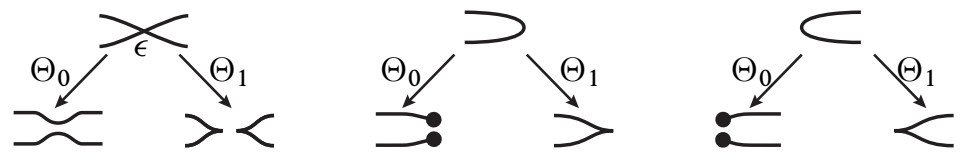

Figure 4: Local splittings

Definition 2.1 (1) We extend the notion of OMS-divide: a cuspidal divide $\Gamma:=$ $(\Gamma, \epsilon)$ is a signed diagram like an OMS-divide except that it has a finite number of horizontal cusps (as in the result of type $\Theta_{1}$ splittings). For instance, a partially (or totally) transformed OMS-divide through $\Theta_{0}$ and $\Theta_{1}$ is a cuspidal divide.

(2) Let $(\Gamma, \epsilon)$ be an OMS-divide (or more generally a cuspidal divide) with double and vertical tangent points numbered by $p_{1}, \ldots, p_{n}$. Let $[k]$ be the word $k_{1} k_{2} \cdots k_{n}, k_{i} \in\{0,1\}$. A state $\left(S, \Theta_{[k]}\right)$ of $(\Gamma, \epsilon)$ is the combination of

- a succession of local splittings $\Theta_{[k]}=\left(\Theta_{k_{1}}, \ldots, \Theta_{k_{n}}\right)$ at $p_{1}, \ldots, p_{n}$;

- the cuspidal divide $S=\Theta_{[k]}(\Gamma, \epsilon)$ without double points nor vertical tangent points obtained by transforming $\Gamma$ through $\Theta_{[k]}$.

For simplification, we will often identify the cuspidal divide $S$ with the state $\left(S, \Theta_{[k]}\right)$. We denote by $\operatorname{St}(\Gamma, \epsilon)$ the set of all states of $(\Gamma, \epsilon)$.

One can define a $j$-strongly invertible link $\mathcal{L}(\Gamma, \epsilon)$ associated with a cuspidal divide $(\Gamma, \epsilon)$ exactly in the same way we have done for OMS-divide. However, such a link is generally unoriented precisely because of the introduction of cusps. Each local splitting at a double point of $(\Gamma, \epsilon)$ corresponds to simultaneously smoothing two symmetric crossing points of the corresponding representative closed braid diagram of $\mathcal{L}(\Gamma, \epsilon)$ [3] whereas each local splitting at a vertical tangent point corresponds to smoothing a crossing point through the axis of the inversion $j$ (see Figure 5).

Let $\Gamma:=(\Gamma, \epsilon)$ be an OMS-divide. Let $n=n_{+}+n_{-}+n_{0}$ be the number of singular points of $\Gamma$ where $n_{+}, n_{-}$are respectively the numbers of positive and negative double points, and $n_{0}$ the number of vertical tangent points. Let's call

$$
w(\Gamma)=2 n_{+}-2 n_{-}+n_{0}
$$

the writhe of $\Gamma$ (ie the writhe of the representative closed braid diagram of $\mathcal{L}(\Gamma, \epsilon)$ [3] with $2 n_{+}+2 n_{-}+n_{0}$ crossings obtained from $(\Gamma, \epsilon)$ ). 


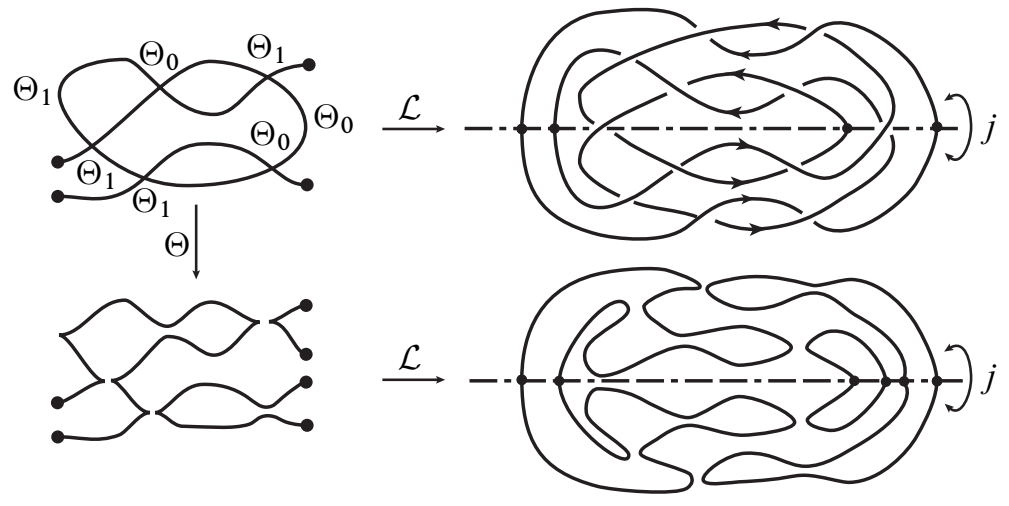

Figure 5: The links $\mathcal{L}(\Gamma, \epsilon)$ and $\mathcal{L}(\Theta(\Gamma, \epsilon)), \Theta=\Theta_{1110010}$

For a state $S \in \operatorname{St}(\Gamma, \epsilon)$, let $\operatorname{cl}(S)$ be the number of closed connected components and $\operatorname{op}(S)$ be the number of open connected components (ie with two end points). Let $r_{+}(S), r_{-}(S)$ and $r_{0}(S)$ be the numbers of $\Theta_{1}$ local splittings (see Figure 4) for positive double points, negative double points and vertical tangent points respectively to obtain $S$ from $(\Gamma, \varepsilon)$. Let's set

$$
i(S)=r_{+}(S)-r_{-}(S)+r_{0}(S), \quad k(S)=w(\Gamma)+2 i(S)-r_{0}(S) .
$$

Definition 2.2 ( $\operatorname{cf}[2]$ ) The polynomial of an OMS-divide $\Gamma$ (and more generally of a cuspidal divide) is the Laurent polynomial (of the variable $\sqrt{t}$ ) defined by:

$$
W_{\Gamma}(t)=\sum_{S \in \operatorname{St}(\Gamma, \epsilon)}(-1)^{i(S)}(\sqrt{t})^{k(S)}\left(\frac{1}{t}+t\right)^{\mathrm{cl}(S)}\left(\frac{1}{\sqrt{t}}+\sqrt{t}\right)^{\mathrm{op}(S)-1}
$$

Proposition 2.3 (cf [2]) The polynomial of an OMS-divide is invariant under $\mathcal{M}-$ equivalence of OMS-divides and so is an invariant for strong equivalence of strongly invertible links.

Definition 2.4 A state $S$ with + or - assignment to each connected component is called an enhanced state, and is denoted by $\widetilde{S}$. The set of enhanced states of $(\Gamma, \epsilon)$ is denoted by $\widetilde{\operatorname{St}}(\Gamma, \epsilon)$, and $S$ is called the underlying state of $\widetilde{S}$.

Let $\widetilde{S}$ be an enhanced state with underling state $S$. The numbers $i(\widetilde{S}):=i(S)$ and $k(\widetilde{S}):=k(S)$ in $(2-2)$ do not depend on the signs of the components. The subset of enhanced states $\widetilde{S}$ of $(\Gamma, \epsilon)$ such that $i(\widetilde{S})=i$ is denoted by $\widetilde{\operatorname{St}}_{i}(\Gamma, \epsilon)$. 
Let's denote by $\delta_{\mathrm{cl}}(\widetilde{S})$ (resp. $\left.\delta_{\mathrm{op}}(\tilde{S})\right)$ the difference between the number of positive and negative closed (resp. open) components of $\widetilde{S}$. Then we define the degree $j(\widetilde{S})$ of the enhanced state $\widetilde{S}$, which depends on the signs of the components of $S$ by

$$
j(\widetilde{S})=k(\tilde{S})+2 \delta_{\mathrm{cl}}(\widetilde{S})+\delta_{\mathrm{op}}(\widetilde{S}) .
$$

We can now reformulate the polynomial of an OMS-divide $\Gamma$ :

$$
W_{\Gamma}(t)=\frac{\sqrt{t}}{1+t} \sum_{\widetilde{S} \in \widetilde{\operatorname{St}}(\Gamma)}(-1)^{i(\tilde{S})}(\sqrt{t})^{j(\tilde{S})}
$$

Remark 2.5 $j(\tilde{S})$ always has the same parity as half the number of end points of $\Gamma$. We also have the inequalities:

$$
-n_{-} \leq i(\tilde{S}) \leq n_{+}+n_{0}, \quad 2 n_{+}-4 n_{-}+n_{0} \leq k(\widetilde{S}) \leq 4 n_{+}-2 n_{-}+2 n_{0} .
$$

\section{Categorification}

\subsection{Complex associated with an OMS-divide}

In this section, we define a graded complex of $\mathbb{Z}_{2}$-vector spaces ${ }^{2}$ associated with a divide. We follow here Viro's approach of Khovanov homology for links [5], based on the Kauffman state model for the Jones polynomial: the polynomial of a divide also have been defined in [2] by state model.

Let $\Gamma:=(\Gamma, \epsilon)$ be an OMS-divide (or a cuspidal divide). For $i \in \mathbb{Z}$, let $\llbracket \Gamma \rrbracket_{i}=$ $\mathbb{Z}_{2}\left\{\widetilde{\mathrm{St}}_{i}(\Gamma)\right\}$ be the finite dimensional $\mathbb{Z}_{2}$-vector space generated by enhanced states $\tilde{S}$ of $\Gamma$ such that $i(\tilde{S})=i$ (if $i<-n_{-}$or $i>n_{+}+n_{0}, \llbracket \Gamma \rrbracket_{i}=\{0\}$ ). Degree $j(\widetilde{S})$ defines a grading on $\llbracket \Gamma \rrbracket_{i}$ and we denote

$$
\llbracket \Gamma \rrbracket=\left(\llbracket \Gamma \rrbracket_{i}\right)_{i \in \mathbb{Z}} \quad \llbracket \Gamma \rrbracket_{i}=\bigoplus_{j \in \mathbb{Z}} \llbracket \Gamma \rrbracket_{i, j}
$$

where

$$
\llbracket \Gamma \rrbracket_{i, j}=\mathbb{Z}_{2}\left\{\tilde{S} \in \mathrm{St}_{i}(\Gamma): j(\widetilde{S})=j\right\} .
$$

Now we define a differential on $\llbracket \Gamma \rrbracket$ to obtain a (finite) complex of graded $\mathbb{Z}_{2}$-vector spaces.

\footnotetext{
${ }^{2}$ Here we choose $\mathbb{Z}_{2}$-vector spaces for simplification to avoid signs. One can easily generalize taking for instance $\mathbb{Z}$-modules or $\mathbb{Q}$-vector spaces.
} 
Definition 3.1 Let $\widetilde{S}_{1}, \widetilde{S}_{2} \in \widetilde{\operatorname{St}}(\Gamma, \epsilon)$. We say that $\widetilde{S}_{2}$ is adjacent to $\widetilde{S}_{1}\left(\widetilde{S}_{1} \rightsquigarrow \widetilde{S}_{2}\right)$ if:

(1) $S_{1}$ and $S_{2}$ coincide outside a neighborhood $D_{p}$ of a singular point $p$ of $(\Gamma, \epsilon)$;

(2) One can pass from $\widetilde{S}_{1}$ to $\widetilde{S}_{2}$ by one of the following transformations $T$ in $D_{p}$ : - $T=\Theta_{1} \circ \Theta_{0}^{-1}$ if $p$ is a positive double point or a vertical tangent point of $(\Gamma, \epsilon)$;

- $T=\Theta_{0} \circ \Theta_{1}^{-1}$ if $p$ is a negative double point;

(3) Signs rules described in Figures 6, 7, 8 are fulfilled, signs of other components being unchanged. (In these figures, black color is used for open components and gray for closed components, a dotted line means that the points are related in the state outside $D_{p}$. Lack of dotted line means that the points are not related outside $D_{p}$ ).

If $\widetilde{S}_{2}$ is adjacent to $\widetilde{S}_{1}$ then

$$
j\left(\widetilde{S}_{1}\right)=j\left(\widetilde{S}_{2}\right), \quad i\left(S_{1}\right)=i\left(S_{2}\right)-1 .
$$

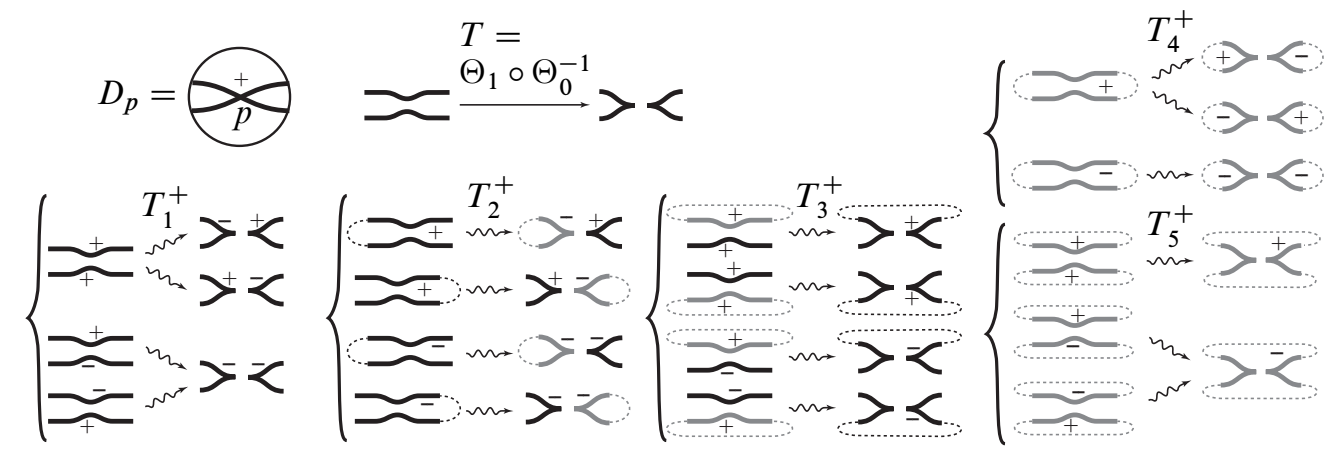

Figure 6: Case of a splitting at a positive double point $p(\epsilon(p)=+)$

The differential $d=\left(d_{i}\right)_{i \in \mathbb{Z}}$ on $\llbracket \Gamma \rrbracket, d_{i}: \llbracket \Gamma \rrbracket_{i} \rightarrow \llbracket \Gamma \rrbracket_{i+1}$ is now defined in the following way: the matrix of $d_{i}$ has coefficients defined by the incidence numbers $\left(\widetilde{S}_{1}: \widetilde{S}_{2}\right), \widetilde{S}_{1} \in{\widetilde{\mathrm{St}_{i}}}_{i}(\Gamma), \widetilde{S}_{2} \in \widetilde{\mathrm{St}}_{i+1}(\Gamma)$ :

$$
\left(\widetilde{S}_{1}: \widetilde{S}_{2}\right)= \begin{cases}1 & \text { if } \widetilde{S}_{1} \rightsquigarrow \widetilde{S}_{2}, \\ 0 & \text { else. }\end{cases}
$$

From (3-2), $d$ respects the degree $j$, ie

$$
d_{i}=\bigoplus_{j} d_{i, j}: \bigoplus_{j} \llbracket \Gamma \rrbracket_{i, j} \rightarrow \bigoplus_{j} \llbracket \Gamma \rrbracket_{i+1, j}
$$




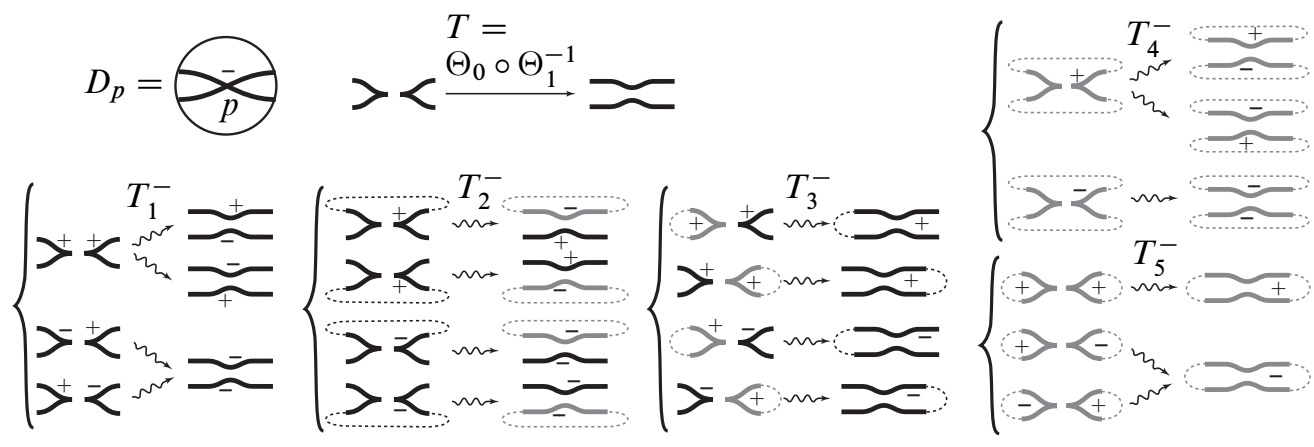

Figure 7: Case of a splitting at a negative double point $p(\epsilon(p)=-)$

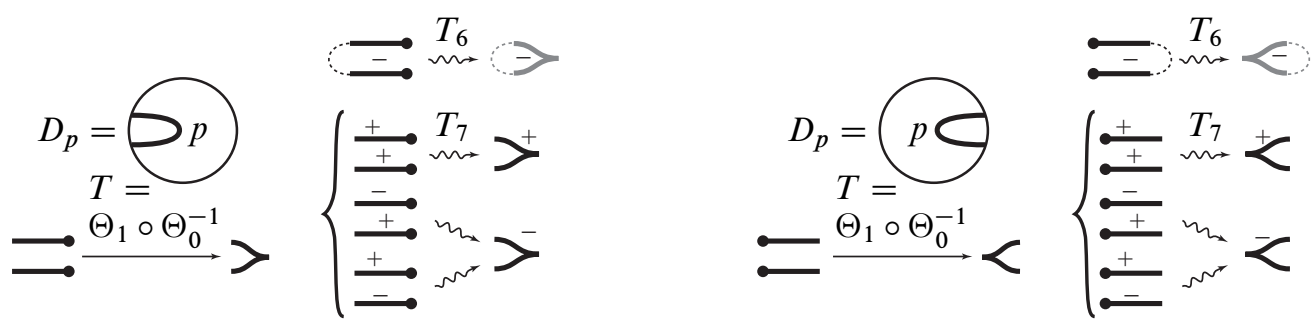

Figure 8: Case of a splitting at a vertical tangent point $p$

Remark 3.2 We have dual roles for $T_{1}^{+}$and $T_{1}^{-}, T_{2}^{+}$and $T_{3}^{-}, T_{3}^{+}$and $T_{2}^{-}, T_{4}^{+}$ and $T_{5}^{-}, T_{5}^{+}$and $T_{4}^{-}$in Figure 6 and Figure 7. To go further about duality property, we could have introduced "negative tangent points" to interpret dual arrows of $T_{6}$ and $T_{7}$ in Figure 8. However, we didn't choose this option, since such "negative tangent points" can be replaced by:

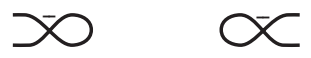

Also, we can see that $T_{i}^{+}$and $T_{i}^{-}, i \in\{1,2,3,4\}$ give rise to analogous situations.

Proposition 3.3 The complex

$$
(\llbracket \Gamma \rrbracket, d)=\left(\llbracket \Gamma \rrbracket_{i}, d_{i}\right)_{i \in \mathbb{Z}}=\left(\bigoplus_{j \in \mathbb{Z}} \llbracket \Gamma \rrbracket_{i, j}, \bigoplus_{j \in \mathbb{Z}} d_{i, j}\right)_{i \in \mathbb{Z}}
$$

is a finite complex of graded $\mathbb{Z}_{2}$-vector spaces (each $\llbracket \Gamma \rrbracket_{i}$ is finitely graded by degree $j$ ). 
Proof It suffices to verify all such diagrams

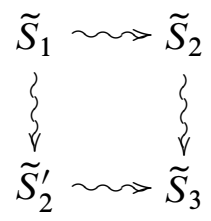

corresponding to splitting two singular points are commutative. Since we have $\mathbb{Z}_{2}-$ vector spaces, commutative diagrams induce the relations $d_{i+1} d_{i}=0$. Notice that from the previous remark, we can strongly reduce the number of cases to check (see also the proof of Proposition 3.5).

\subsection{Alternative point of view}

Here we present an alternative (more algebraic) way to see the complex $(\llbracket \Gamma \rrbracket, d)$, in terms of Frobenius algebra: we can link complexes of OMS-divides with $1+1-\mathrm{TQFT}$ (or more precisely with some $1+1-\mathrm{TQFT}$ with symmetry property).

Let $\mathcal{A}:=\mathbb{Z}_{2}\left\{v_{-}, v_{+}\right\}$be the graded $\mathbb{Z}_{2}$-vector space generated by two elements $v_{-}$ and $v_{+}$such that $\operatorname{deg}\left(v_{-}\right)=-1$ and $\operatorname{deg}\left(v_{+}\right)=1$. We define a commutative product $\mu_{1}: \mathcal{A} \otimes \mathcal{A} \rightarrow \mathcal{A}$, a unit $\eta_{1}: \mathbb{Z}_{2} \rightarrow \mathcal{A}$ and a nondegenerate symmetric bilinear pairing $\beta_{1}: \mathcal{A} \otimes \mathcal{A} \rightarrow \mathbb{Z}_{2}$ by:

$$
\begin{array}{ll} 
& \mu_{1}\left(v_{+} \otimes v_{+}\right)=v_{+}, \quad \mu_{1}\left(v_{+} \otimes v_{-}\right)=\mu_{1}\left(v_{-} \otimes v_{+}\right)=v_{-}, \quad \mu_{1}\left(v_{-} \otimes v_{-}\right)=0 \\
(3-5) \quad & \eta_{1}(0)=0, \quad \eta_{1}(1)=v_{+} \\
& \beta_{1}\left(v_{+} \otimes v_{+}\right)=0, \quad \beta_{1}\left(v_{+} \otimes v_{-}\right)=\beta_{1}\left(v_{-} \otimes v_{+}\right)=1, \quad \beta_{1}\left(v_{-} \otimes v_{-}\right)=0 .
\end{array}
$$

The form $\beta_{1}$ induces a duality isomorphism $\mathcal{A} \stackrel{\simeq}{\longleftrightarrow} \mathcal{A}^{*}$ and $\mathcal{A}$ is a commutative Frobenius algebra with adjoint coproduct $\delta_{1}: \mathcal{A} \rightarrow \mathcal{A} \otimes \mathcal{A}$ and counit $\varepsilon_{1}: \mathcal{A} \rightarrow \mathbb{Z}_{2}$ :

$$
\begin{array}{ll}
\delta_{1}\left(v_{+}\right)=v_{+} \otimes v_{-}+v_{-} \otimes v_{+} & \varepsilon_{1}\left(v_{+}\right)=0 \\
\delta_{1}\left(v_{-}\right)=v_{-} \otimes v_{-} & \varepsilon_{1}\left(v_{-}\right)=1 .
\end{array}
$$

Let $\phi_{1}: \mathcal{A} \otimes \mathcal{A} \rightarrow \mathcal{A} \otimes \mathcal{A}$ be the flip morphism: $\phi_{1}\left(a \otimes a^{\prime}\right)=a^{\prime} \otimes a$, and $A: \mathcal{A} \rightarrow \mathcal{A}$ the identity morphism. The morphisms $\mu_{1}, \delta_{1}, \eta_{1}, \varepsilon_{1}$ are homogeneous with respective 
degrees $-1,-1,1,1$ and satisfy the relations of associativity, commutativity, coassociativity, co-commutativity:

$$
\left\{\begin{array} { l } 
{ \mu _ { 1 } \circ \phi _ { 1 } = \mu _ { 1 } } \\
{ \mu _ { 1 } \circ ( \mu _ { 1 } \otimes A ) = \mu _ { 1 } \circ ( A \otimes \mu _ { 1 } ) } \\
{ \mu _ { 1 } \circ ( \eta _ { 1 } \otimes A ) = A }
\end{array} \quad \left\{\begin{array}{l}
\phi_{1} \circ \delta_{1}=\delta_{1} \\
\left(\delta_{1} \otimes A\right) \circ \delta_{1}=\left(A \otimes \delta_{1}\right) \circ \delta_{1} \\
\left(\varepsilon_{1} \otimes A\right) \circ \delta_{1}=\left(A \otimes \varepsilon_{1}\right) \circ \delta_{1}=A
\end{array}\right.\right.
$$

and

$$
\delta_{1} \circ \mu_{1}=\left(\mu_{1} \otimes A\right) \circ\left(A \otimes \delta_{1}\right) .
$$

The vector space $\mathcal{A} \otimes \mathcal{A}$ has an induced structure of graded commutative Frobenius algebra with product, coproduct, unit and co-unit:

$$
\begin{array}{ll}
\mu_{1}^{\otimes}=\left(\mu_{1} \otimes \mu_{1}\right) \circ(A \otimes \phi \otimes A) & \eta_{1}^{\otimes}=\eta_{1} \otimes \eta_{1} \\
\delta_{1}^{\otimes}=(A \otimes \phi \otimes A) \circ\left(\delta_{1} \otimes \delta_{1}\right) & \varepsilon_{1}^{\otimes}=\varepsilon_{1} \otimes \varepsilon_{1} .
\end{array}
$$

Let $\mathcal{B}:=\mathbb{Z}_{2}\left\{w_{-}, w_{+}\right\}$be the graded $\mathbb{Z}_{2}$-vector space generated by two elements $w_{-}$ and $w_{+}, \operatorname{deg}\left(w_{-}\right)=-2, \operatorname{deg}\left(w_{+}\right)=2$. Let's consider respectively the injection and surjection $l: \mathcal{B} \rightarrow \mathcal{A} \otimes \mathcal{A}$ and $\pi: \mathcal{A} \otimes \mathcal{A} \rightarrow \mathcal{B}$ defined by:

$$
\begin{array}{ll}
l\left(w_{+}\right)=v_{+} \otimes v_{+} & \pi\left(v_{+} \otimes v_{+}\right)=w_{+} \\
l\left(w_{-}\right)=v_{-} \otimes v_{-} & \pi\left(v_{+} \otimes v_{-}\right)=\pi\left(v_{-} \otimes v_{+}\right)=0 \\
& \pi\left(v_{-} \otimes v_{-}\right)=w_{-} .
\end{array}
$$

Then $\mathcal{B}$ canonically inherits from $\mathcal{A} \otimes \mathcal{A}$ of a structure of graded commutative Frobenius algebra with product, coproduct, unit and co-unit $\mu_{2}, \delta_{2}, \eta_{2}, \varepsilon_{2}$ with respective degrees $-2,-2,2,2$ satisfying

(3-10) $\mu_{2}=\pi \circ \mu_{1}^{\otimes} \circ(l \otimes l) \quad \delta_{2}=(\pi \otimes \pi) \circ \delta_{1}^{\otimes} \circ l \quad \eta_{2}=\pi \circ \eta_{1}^{\otimes} \quad \varepsilon_{2}=\varepsilon_{1}^{\otimes} \circ l$.

The morphisms $l$ and $\pi$ are adjoint with degree -2 . We denote by $\phi_{2}: \mathcal{B} \otimes \mathcal{B} \rightarrow \mathcal{B} \otimes \mathcal{B}$ the flip morphism $\phi_{2}\left(b \otimes b^{\prime}\right)=b^{\prime} \otimes b$, and $B: \mathcal{B} \rightarrow \mathcal{B}$ the identity morphism.

For each (nonenhanced) state $S$ of $\Gamma$, let's number all open components with $p \in$ $\{1, \ldots, o p(S)\}$ and all closed components with $q \in\{1, \ldots, \operatorname{cl}(S)\}$. Then for an enhanced state $\widetilde{S}$ with underlying state $S$,we define the tensor product

$$
t(\tilde{S})=\bigotimes_{p=1}^{\mathrm{op}(S)} v_{ \pm_{\mathrm{op}}(p)} \otimes \bigotimes_{q=1}^{\mathrm{cl}(S)} w_{ \pm_{\mathrm{cl}}(q)} \in \mathcal{A}^{\otimes \mathrm{op}(S)} \otimes \mathcal{B}^{\otimes \mathrm{cl}(S)}
$$

where $\pm_{\mathrm{op}}(p)$ and $\pm_{\mathrm{cl}}(q)$ are the + or - signs of the $p$-th open and the $q$-th closed components of $\tilde{S}$ respectively. The degree of $t(\tilde{S})$ does not correspond to the degree $j(\widetilde{S})$ of $\widetilde{S}$ :

$$
\operatorname{deg}(t(\tilde{S}))=\delta_{\mathrm{op}}(\widetilde{S})+2 \delta_{\mathrm{cl}}(\widetilde{S})=j(\widetilde{S})-k(\widetilde{S})=j(\widetilde{S})-k(S) .
$$


So we introduce the following definition:

Definition 3.4 (Translation of the degree of a graded vector space) Let $\mathcal{V}=\bigoplus_{j \in \mathbb{Z}} \mathcal{V}_{j}$ be a graded $\mathbb{Z}_{2}$-vector space. The translated graded $\mathbb{Z}_{2}$-vector space $\mathcal{V}\{\ell\}$ is defined by $\mathcal{V}\{\ell\}_{j}=\mathcal{V}_{j-\ell}$.

Now we translate the degree of $t(\widetilde{S})$ by $k(S)$ and we define

$$
\mathcal{C}(\Gamma)=\left(\mathcal{C}_{i}(\Gamma)\right)_{i \in \mathbb{Z}} \quad \text { where } \mathcal{C}_{i}(\Gamma)=\bigoplus_{\substack{S \in \operatorname{St}(\Gamma) \\ i(S)=i}}\left(\mathcal{A}^{\otimes \mathrm{op}(S)} \otimes \mathcal{B}^{\otimes \mathrm{cl}(S)}\right)\{k(S)\}
$$

Proposition 3.5 The map $t: \widetilde{\operatorname{St}}(\Gamma) \rightarrow \mathcal{C}(\Gamma)$ defined by (3-11) extends to an isomorphism of complexes: $t: \llbracket \Gamma \rrbracket \rightarrow \mathcal{C}(\Gamma)$.

Proof The incidence relations $T_{i}^{ \pm}, 1 \leq i \leq 5$ of Figures 6,7 induce morphisms of $\mathbb{Z}_{2}$-vector spaces denoted by $T_{i}$, which have degree -2 :

$$
\begin{array}{lll}
T_{1}=\delta_{1} \circ \mu_{1} & T_{3}=\mu_{1} \circ\left(A \otimes \mu_{1}\right) \circ(A \otimes \imath) & T_{5}=\mu_{2} \\
T_{2}=(A \otimes \pi) \circ\left(A \otimes \delta_{1}\right) \circ \delta_{1} & T_{4}=\delta_{2} . &
\end{array}
$$

The incidence relations $T_{6}, T_{7}$ of Figure 8 induce morphisms denoted by the same letters $T_{6}, T_{7}$, which have degree -1 :

$$
T_{6}=\pi \circ \delta_{1} \quad T_{7}=\mu_{1} .
$$

More precisely, we have:

$$
\begin{aligned}
& T_{1}: \mathcal{A} \otimes \mathcal{A} \rightarrow \mathcal{A} \otimes \mathcal{A} \\
& v_{+} \otimes v_{+} \mapsto\left\{\begin{array}{l}
v_{+} \otimes v_{-} \\
+v_{-} \otimes v_{+}
\end{array}\right. \\
& \left.\begin{array}{ll}
v_{+} \otimes & v_{-} \\
v_{-} \otimes & v_{+}
\end{array}\right\} \mapsto v_{-} \otimes v_{-} \\
& v_{-} \otimes v_{-} \mapsto 0 \\
& T_{3}: \mathcal{A} \otimes \mathcal{B} \quad \rightarrow \mathcal{A} \quad T_{5}: \quad \mathcal{B} \otimes \mathcal{B} \quad \rightarrow \mathcal{B} \\
& v_{+} \otimes w_{+} \mapsto v_{+} \quad w_{+} \otimes w_{+} \mapsto w_{+} \\
& v_{-} \otimes w_{+} \mapsto v_{-} \\
& \left.\begin{array}{cc}
v_{+} \otimes & w_{-} \\
v_{-} \otimes & w_{-}
\end{array}\right\} \mapsto 0 \\
& \left.\begin{array}{l}
w_{+} \otimes w_{-} \\
w_{-} \otimes w_{+}
\end{array}\right\} \mapsto w_{-} \\
& w_{-} \otimes w_{-} \mapsto 0 \\
& T_{2}: \mathcal{A} \rightarrow \mathcal{A} \otimes \mathcal{B} \\
& v_{+} \mapsto v_{+} \otimes w_{-} \\
& v_{-} \mapsto v_{-} \otimes w_{-} \\
& T_{4}: \mathcal{B} \rightarrow \mathcal{B} \otimes \mathcal{B} \\
& w_{+} \mapsto w_{+} \otimes w_{-}+w_{-} \otimes w_{+} \\
& w_{-} \mapsto w_{-} \otimes w_{-} \\
& T_{7}: \mathcal{A} \otimes \mathcal{A} \rightarrow \mathcal{A} \\
& T_{6}: \mathcal{A} \rightarrow \mathcal{B} \\
& v_{+} \mapsto 0 \\
& v_{-} \mapsto w_{-} \\
& v_{+} \otimes v_{+} \mapsto v_{+} \\
& \left.\begin{array}{l}
v_{+} \otimes v_{-} \\
v_{-} \otimes v_{+}
\end{array}\right\} \mapsto v_{-} \\
& v_{-} \otimes v_{-} \mapsto 0
\end{aligned}
$$


Using these morphisms, we transfer the differential on $\llbracket \Gamma \rrbracket$ to a differential on $\mathcal{C}(\Gamma)$. Notice according to Remark 3.2 that $T_{1}$ is self-adjoint and that $T_{2}$ and $T_{3}$ (resp. $T_{4}$ and $T_{5}$ ) are adjoint. Moreover, $T_{2}$ and $T_{4}$ are injective whereas $T_{3}$ and $T_{5}$ are surjective. Also $T_{7}$ is surjective. The relation $d \circ d=0$ (induced by commutative diagrams (3-4) in the proof of Proposition 3.3) is recovered using the following relations:

- Symmetry properties:

$$
\begin{array}{ll}
T_{1} \circ \phi_{1}=\phi_{1} \circ T_{1}=T_{1} & T_{4}=\phi_{2} \circ T_{4} \\
\left(A \otimes T_{2}\right) \circ \phi_{1}=\left(\phi_{1} \otimes B\right) \circ\left(A \otimes T_{2}\right) & T_{5}=T_{5} \circ \phi_{2} \\
\phi_{1} \circ\left(A \otimes T_{3}\right)=\left(A \otimes T_{3}\right) \circ\left(\phi_{1} \otimes B\right) & T_{7}=T_{7} \circ \phi_{1}
\end{array}
$$

- Commutativity properties corresponding to the splitting of two double points:

$$
\begin{array}{cc}
\left(T_{1} \otimes A\right) \circ\left(A \otimes T_{1}\right)=\left(A \otimes T_{1}\right) \circ\left(T_{1} \otimes A\right) & \left(T_{4} \otimes B\right) \circ T_{4}=\left(B \otimes T_{4}\right) \circ T_{4} \\
\left(A \otimes T_{2}\right) \circ T_{1}=\left(T_{1} \otimes B\right) \circ\left(A \otimes T_{2}\right) & T_{5} \circ\left(T_{5} \otimes B\right)=T_{5} \circ\left(B \otimes T_{5}\right) \\
T_{1} \circ\left(A \otimes T_{3}\right)=\left(A \otimes T_{3}\right) \circ\left(T_{1} \otimes B\right) & T_{4} \circ T_{5}=\left(B \otimes T_{5}\right) \circ\left(T_{4} \otimes B\right) \\
T_{1} \circ T_{1}=\left(A \otimes T_{3}\right) \circ\left(\phi_{1} \otimes B\right) \circ\left(A \otimes T_{2}\right)=0 \\
\left(T_{2} \otimes B\right) \circ T_{2}=\left(A \otimes \phi_{2}\right) \circ\left(T_{2} \otimes B\right) \circ T_{2}=\left(A \otimes T_{4}\right) \circ T_{2} \\
T_{3} \circ\left(T_{3} \otimes B\right)=T_{3} \circ\left(T_{3} \otimes B\right) \circ\left(A \otimes \phi_{2}\right)=T_{3} \circ\left(A \otimes T_{5}\right) \\
T_{2} \circ T_{3}=\left(T_{3} \otimes B\right) \circ\left(A \otimes \phi_{2}\right) \circ\left(T_{2} \otimes B\right) \\
=\left(T_{3} \otimes B\right) \circ\left(A \otimes T_{4}\right)=\left(A \otimes T_{5}\right) \circ\left(T_{2} \otimes B\right)
\end{array}
$$

- Commutativity properties corresponding to the splitting of a double point and a vertical tangent point:

$$
\begin{array}{ll}
T_{4} \circ T_{6}=\left(T_{6} \otimes B\right) \circ T_{2} & T_{7} \circ\left(A \otimes T_{3}\right)=T_{3} \circ\left(T_{7} \otimes B\right) \\
T_{6} \circ T_{3}=T_{5} \circ\left(T_{6} \otimes B\right) & T_{2} \circ T_{7}=\left(T_{7} \otimes B\right) \circ\left(A \otimes T_{2}\right) \\
T_{2} \circ T_{7}=\left(A \otimes T_{6}\right) \circ T_{1} & T_{7} \circ T_{1}=0=T_{3} \circ\left(A \otimes T_{6}\right)
\end{array}
$$

- Commutativity properties corresponding to the splitting of two vertical tangent points:

$$
T_{1} \circ\left(A \otimes T_{7}\right)=\left(A \otimes T_{7}\right) \circ\left(T_{1} \otimes A\right) .
$$

Remark 3.6 The units and counits $\eta_{1}, \eta_{2}, \varepsilon_{1}, \varepsilon_{2}$ of $\mathcal{A}$ and $\mathcal{B}$ correspond respectively to the creation of a positive open component, the creation of a positive closed component, the destruction of a negative open component and the destruction of a negative closed component. Besides, $A \otimes \varepsilon_{2}$ and $B \otimes \varepsilon_{2}$ are left inverses of $T_{2}$ and $T_{4}$ whereas 
$A \otimes \eta_{2}, B \otimes \eta_{2}$ and $A \otimes \eta_{1}$ are right inverses of $T_{3}, T_{5}$ and $T_{7}$. In Section 4, we will often refer to these morphisms and to the following ones:

$$
\begin{array}{rlrlrlrl}
\bar{\eta}_{1}: \mathbb{Z}_{2} & \rightarrow \mathcal{A} & \bar{\eta}_{2}: \mathbb{Z}_{2} & \rightarrow \mathcal{B} & \bar{\varepsilon}_{1}: \mathcal{A} \rightarrow \mathbb{Z}_{2} & \bar{\varepsilon}_{2}: \mathcal{B} \rightarrow \mathbb{Z}_{2} \\
1 & \mapsto v_{-} & 1 \mapsto w_{-} & v_{+} \mapsto 1 & w_{+} \mapsto 1 \\
& & v_{-} \mapsto 0 & & w_{-} \mapsto 0
\end{array}
$$

which correspond respectively to the creation of a negative open component, the creation of a negative closed component, the destruction of a positive open component and the destruction of a positive closed component, and to the following composed morphisms:

$$
\begin{array}{rlrl}
\tau=\eta_{1} \varepsilon_{1}: \mathcal{A} & \rightarrow \mathcal{A} & \sigma=\bar{\eta}_{1} \varepsilon_{2}: \mathcal{B} & \rightarrow \mathcal{A} \\
v_{+} & \mapsto 0 & w_{+} \mapsto 0 \\
v_{-} & \mapsto v_{+} & w_{-} \mapsto v_{-}
\end{array}
$$

\subsection{Review of basic facts about complexes}

Let $\mathcal{C}:=(\mathcal{C}, d)=\left(\mathcal{C}_{i}, d_{i}\right)_{i \in \mathbb{Z}}$ be a complex of $\mathbb{Z}_{2}$-vector spaces. We denote by $\mathcal{H}(\mathcal{C})$ its homology

$$
\mathcal{H}(\mathcal{C})=\left(H_{i}\right)_{i \in \mathbb{Z}}, \quad H_{i}=\operatorname{Ker} d_{i} / \operatorname{Im} d_{i-1} .
$$

A complex is acyclic if its homology is null.

Definition 3.7 (Shift of the grading of a complex) Let $(\mathcal{C}, d)=\left(\mathcal{C}_{i}, d_{i}\right)_{i \in \mathbb{Z}}$ be a complex of $\mathbb{Z}_{2}$-vector spaces. We define the complex

$$
(\mathcal{C}, d)[k]=(\mathcal{C}[k], d[k]) \quad \text { by } \quad \mathcal{C}[k]_{i}=\mathcal{C}_{i-k} \quad \text { and } \quad d[k]_{i}=d_{i-k} .
$$

(If $(\mathcal{C}, d)=\left(\bigoplus_{j \in \mathbb{Z}} \mathcal{C}_{i, j}, \bigoplus_{j \in \mathbb{Z}} d_{i, j}\right)_{i \in \mathbb{Z}}$ is a complex of graded $\mathbb{Z}_{2}$-vector spaces, then we can translate both the grading of the complex and the degree of the vector spaces:

$$
(\mathcal{C}, d)[k]\{\ell\}=(\mathcal{C}, d)\{\ell\}[k]
$$

is defined by $\left.\mathcal{C}[k]\{\ell\}_{i, j}=\mathcal{C}_{i-k, j-\ell}, \quad d[k]\{\ell\}_{i, j}=d_{i-k, j-\ell}\right)$.

A morphism of complexes of $\mathbb{Z}_{2}$-vector spaces $f:\left(\mathcal{C}^{0}, d^{0}\right) \rightarrow\left(\mathcal{C}^{1}, d^{1}\right)$ is a sequence $f=\left(f_{i}\right)_{i \in \mathbb{Z}}$ of linear maps $f_{i}: \mathcal{C}_{i}^{0} \rightarrow \mathcal{C}_{i}^{1}$ such that ${ }^{3}: f d^{0}=d^{1} f$ (ie for all $i$, $\left.d_{i}^{1} f_{i}=f_{i+1} d_{i}^{0}\right)$.

\footnotetext{
${ }^{3}$ Since we are working with field $\mathbb{Z}_{2}$, commutativity and anticommutativity coincide so that we have equivalently $d^{1} f+f d^{0}=0$.
} 
Definition 3.8 For a morphism of complexes $f:\left(\mathcal{C}^{0}, d^{0}\right) \rightarrow\left(\mathcal{C}^{1}, d^{1}\right)$, the cone of $f$ is the complex denoted by $\operatorname{Cone}(f)=\left(\mathcal{C}_{i}, D_{i}\right)_{i \in \mathbb{Z}}$ and defined by

$$
\mathcal{C}_{i}=\mathcal{C}_{i}^{0} \oplus \mathcal{C}_{i-1}^{1}=\mathcal{C}_{i}^{0} \oplus\left(\mathcal{C}^{1}[1]\right)_{i}, \quad D_{i}=\left(\begin{array}{cc}
d_{i}^{0} & 0 \\
f_{i} & d_{i-1}^{1}
\end{array}\right) .
$$

(Notice that $\left(\mathcal{C}^{0}, d^{0}\right)$ is a quotient-complex and $\left(\mathcal{C}^{1}, d^{1}\right)[1]$ a subcomplex of $\left.(\mathcal{C}, D)\right)$.

A morphism of complexes $f:\left(\mathcal{C}^{0}, d^{0}\right) \rightarrow\left(\mathcal{C}^{1}, d^{1}\right)$ induces an isomorphism in homology if and only if Cone $(f)$ is acyclic. This is the case if $f$ is a homotopy equivalence of complexes, ie there exist a morphism of complexes $g:\left(\mathcal{C}^{1}, d^{1}\right) \rightarrow\left(\mathcal{C}^{0}, d^{0}\right)$ and sequences $h^{0}=\left(h_{i}^{0}\right)_{i \in \mathbb{Z}}, h^{1}=\left(h_{i}^{1}\right)_{i \in \mathbb{Z}}$ of linear maps (homotopies) $h_{i}^{0}: \mathcal{C}_{i+1}^{0} \rightarrow \mathcal{C}_{i}^{0}$ and $h_{i}^{1}: \mathcal{C}_{i+1}^{1} \rightarrow \mathcal{C}_{i}^{1}$ such that

$$
g f=\mathrm{id}+h^{0} d^{0}+d^{0} h^{0} \quad \text { and } \quad f g=\mathrm{id}+h^{1} d^{1}+d^{1} h^{1} .
$$

(ie $\forall i g_{i} f_{i}=\mathrm{id}+h_{i}^{0} d_{i}^{0}+d_{i-1}^{0} h_{i-1}^{0} \quad$ and $\quad f_{i} g_{i}=\mathrm{id}+h_{i}^{1} d_{i}^{1}+d_{i-1}^{1} h_{i-1}^{1}$ ).

Remark 3.9 As a particular case, if $h^{0}=0$, the complex $\left(\mathcal{C}^{0}, d^{0}\right)$ is called a strong deformation retract of $\left(\mathcal{C}^{1}, d^{1}\right)$, with inclusion map $f$, retraction $g$ and homotopy map $h^{1}$. Besides, up to changing $h^{1}$ to a new homotopy $h$, we can always suppose that $h h=0, h f=0$ and $g h=0$. We will assume these properties are always satisfied in the definition of strong deformation retraction.

Proposition 3.10 Let $\left(\overline{\mathcal{C}}^{1}, \bar{d}^{1}\right)$ be a strong deformation retract of $\left(\mathcal{C}^{1}, d^{1}\right)$ with retraction $r$, inclusion $j$ and homotopy map $h$ such that $h h=0, r h=0, h j=0$. Let $f:\left(\mathcal{C}^{0}, d^{0}\right) \rightarrow\left(\mathcal{C}^{1}, d^{1}\right)$ be a morphism of complexes. Then Cone $(r f)$ is a strong deformation retract of $\operatorname{Cone}(f)$ with

$$
\begin{array}{ll}
\text { retraction } & R=\left(\begin{array}{cc}
\text { id } & 0 \\
0 & r
\end{array}\right), \\
\text { inclusion } & J=\left(\begin{array}{cc}
\text { id } & 0 \\
h f & j
\end{array}\right), \\
\text { homotopy } & H=\left(\begin{array}{ll}
0 & 0 \\
0 & h
\end{array}\right)
\end{array}
$$

such that $H H=0, R H=0$ and $H J=0$.

Proof Immediate. 
A double complex $(\mathcal{C}, d, \partial)$ is a sequence of complexes $\left(\mathcal{C}^{k}, d^{k}\right)_{k \in \mathbb{Z}}$ of $\mathbb{Z}_{2}$-vector spaces and morphisms of complexes $\left(\partial^{k}\right)_{k \in \mathbb{Z}}$ :

$$
\cdots \stackrel{\partial^{k-1}}{\longrightarrow}\left(\mathcal{C}^{k}, d^{k}\right) \stackrel{\partial^{k}}{\longrightarrow}\left(\mathcal{C}^{k+1}, d^{k+1}\right) \stackrel{\partial^{k+1}}{\longrightarrow}\left(\mathcal{C}^{k+2}, d^{k+2}\right) \stackrel{\partial^{k+2}}{\longrightarrow} \cdots
$$

such that for all $k \in \mathbb{Z}, \partial^{k+1} \partial^{k}=0$. A morphism of two double complexes is a sequence of morphisms of complexes $f=\left(f^{k}\right)_{k \in \mathbb{Z}}$ :

$$
\begin{gathered}
\cdots \stackrel{\bar{\partial}^{k-1}}{\longrightarrow}\left(\overline{\mathcal{C}}^{k}, \bar{d}^{k}\right) \stackrel{\bar{\partial}^{k}}{\longrightarrow}\left(\overline{\mathcal{C}}^{k+1}, \bar{d}^{k+1}\right) \stackrel{\bar{\partial}^{k+1}}{\longrightarrow}\left(\overline{\mathcal{C}}^{k+2}, \bar{d}^{k+2}\right) \stackrel{\bar{\partial}^{k+2}}{\longrightarrow} \cdots \\
\qquad f^{k} \\
\cdots \stackrel{\partial^{k-1}}{\longrightarrow}\left(\mathcal{C}^{k}, d^{k}\right) \stackrel{\partial^{k}}{\longrightarrow}\left(\overline{\mathcal{C}}^{k+1}, d^{k+1}\right) \stackrel{\partial^{k+2}}{\longrightarrow}\left(\mathcal{C}^{k+2}, d^{k+2}\right) \stackrel{\partial^{n-1}}{\longrightarrow} \cdots
\end{gathered}
$$

such that for all $k \in \mathbb{Z}, f^{k+1} \bar{\partial}^{k}=\partial^{k} f^{k}$. We also have notions of homotopy equivalence and strong deformation retraction for double complex. A morphism of double complexes $f:(\overline{\mathcal{C}}, \bar{d}, \bar{\partial}) \rightarrow(\mathcal{C}, d, \partial)$ is a homotopy equivalence (of double complexes $)$ if there exists a morphism of double complexes $g:(\mathcal{C}, d, \partial) \rightarrow(\overline{\mathcal{C}}, \bar{d}, \bar{\partial})$ and homotopy maps $\bar{h}=\left(\bar{h}^{k}\right)_{k \in \mathbb{Z}}, h=\left(h^{k}\right)_{k \in \mathbb{Z}}$ (sequences of morphisms of complexes) $\bar{h}^{k}:\left(\overline{\mathcal{C}}^{k+1}, \bar{d}^{k+1}\right) \rightarrow\left(\overline{\mathcal{C}}^{k}, \bar{d}^{k}\right)$ and $\bar{h}^{k}:\left(\mathcal{C}^{k+1}, d^{k+1}\right) \rightarrow\left(\mathcal{C}^{k}, d^{k}\right)$ such that for all $k$

$$
g^{k} f^{k}=\mathrm{id}+\bar{h}^{k} \bar{\partial}^{k}+\bar{\partial}^{k-1} \bar{h}^{k-1} \quad \text { and } \quad f^{k} g^{k}=\mathrm{id}+h^{k} \partial^{k}+\partial^{k-1} h^{k-1} \text {. }
$$

If $\bar{h}=0,(\overline{\mathcal{C}}, \bar{d}, \bar{\partial})$ is called a strong deformation retract of the double complex $(\mathcal{C}, d, \partial)$ with inclusion $f$ and retraction $g$. Again, up to changing the homotopy $h$, we assume that it satisfies: $h h=0, h f=0$ and $g h=0$.

Now we extend the definition of cone to a finite sequence of morphisms of complexes. A double complex $(\mathcal{C}, d, \partial)$ is $\partial$-finite if $\left(\mathcal{C}^{k}, d^{k}\right)$ is trivial except for a finite number of values of $k$.

\section{Definition 3.11 Let}

$$
\left(\mathcal{C}^{0}, d^{0}\right) \stackrel{\partial^{0}}{\longrightarrow} \cdots \stackrel{\partial^{n-1}}{\longrightarrow}\left(\mathcal{C}^{n}, d^{n}\right)
$$

be a $\partial$-finite double complex. Let's denote

$$
\begin{aligned}
\tilde{\partial}_{i}^{0}: \mathcal{C}_{i}^{0} & \rightarrow \mathcal{C}_{i}^{1} \oplus \mathcal{C}_{i-1}^{2} \oplus \cdots \oplus \mathcal{C}_{i-n+1}^{n} \\
u & \mapsto\left(\partial_{i}^{0}(u), 0, \ldots, 0\right) .
\end{aligned}
$$

Then the cone of $\left(\partial^{0}, \ldots, \partial^{n-1}\right)$ is the complex defined by

$$
\operatorname{Cone}\left(\partial^{0}, \ldots, \partial^{n-1}\right)=\operatorname{Cone}\left(\tilde{\partial}^{0}, \operatorname{Cone}\left(\partial^{1}, \ldots, \partial^{n-1}\right)\right) .
$$


Suppose that $f=\left(f^{k}\right)_{0 \leq k \leq n}$ is a morphism from a $\bar{\partial}$-finite double complexes $(\overline{\mathcal{C}}, \bar{d}, \bar{\partial})$ to a $\partial$-finite $(\mathcal{C}, d, \partial)$ :

$$
\left(\overline{\mathcal{C}}^{0}, \bar{d}^{0}\right) \stackrel{\bar{\partial}^{0}}{\longrightarrow} \cdots \stackrel{\bar{\partial}^{n-1}}{\longrightarrow}\left(\overline{\mathcal{C}}^{n}, \bar{d}^{n}\right) \quad \text { and } \quad\left(\mathcal{C}^{0}, d^{0}\right) \stackrel{\partial^{0}}{\longrightarrow} \cdots \stackrel{\partial^{n-1}}{\longrightarrow}\left(\mathcal{C}^{n}, d^{n}\right) .
$$

Let's set $F_{i}=f_{i}^{0} \oplus f_{i-1}^{1} \oplus \cdots \oplus f_{i-n}^{n}$. Then $f$ induces a morphism of complexes

$$
C(f)=\left(F_{i}\right)_{i \in \mathbb{Z}}: \text { Cone }\left(\bar{\partial}^{0}, \ldots, \bar{\partial}^{n-1}\right) \longrightarrow \operatorname{Cone}\left(\partial^{0}, \ldots, \partial^{n-1}\right) .
$$

If $f^{k}$ are isomorphisms, $C(f)$ is also an isomorphism.

Proposition 3.12 If $(\overline{\mathcal{C}}, \bar{d}, \bar{\partial})$ is a $\bar{\partial}$-finite double complex, $(\mathcal{C}, d, \partial)$ a $\partial$-finite double complex and $f:(\overline{\mathcal{C}}, \bar{d}, \bar{\partial}) \rightarrow(\mathcal{C}, d, \partial)$ a homotopy equivalence with inverse $g$, then

$$
C(f): \operatorname{Cone}\left(\bar{\partial}^{0}, \ldots, \bar{\partial}^{n-1}\right) \longrightarrow \operatorname{Cone}\left(\partial^{0}, \ldots, \partial^{n-1}\right)
$$

is a homotopy equivalence of complexes with inverse $C(g)$. So $C(f)$ induces an isomorphism in homology.

Furthermore if $(\overline{\mathcal{C}}, \bar{d}, \bar{\partial})$ is a strong deformation retract of $(\mathcal{C}, d, \partial)$ with inclusion map $f$ and retraction $g$ then Cone $\left(\bar{\partial}^{0}, \ldots, \bar{\partial}^{n-1}\right)$ is a strong deformation retract of Cone $\left(\partial^{0}, \ldots, \partial^{n-1}\right)$ with inclusion map $C(f)$ and retraction $C(g)$, and so $C(f)$ induces an isomorphism in homology.

Proof Let $\bar{h}=\left(\bar{h}^{k}\right)_{1 \leq k<n}$ and $h=\left(h^{k}\right)_{0 \leq k<n}$ be homotopies associated with $f$ and $g$. Then we have:

$$
\begin{aligned}
\bar{h}_{i+1}^{k-1} \bar{d}_{i}^{k}=\bar{d}_{i}^{k-1} \bar{h}_{i}^{k-1} & \bar{h}_{i}^{k} \bar{\partial}_{i}^{k}+\bar{\partial}_{i}^{k-1} \bar{h}_{i}^{k-1}+\mathrm{id}=g_{i}^{k} f_{i}^{k} \\
h_{i+1}^{k-1} d_{i}^{k}=d_{i}^{k-1} h_{i}^{k-1} & h_{i}^{k} \partial_{i}^{k}+\partial_{i}^{k-1} h_{i}^{k-1}+\mathrm{id}=f_{i}^{k} g_{i}^{k}
\end{aligned}
$$

Let $H=\left(H_{i}\right)_{i \in \mathbb{Z}}$ be the sequence of linear maps defined by

$$
\begin{aligned}
H_{i}: \mathcal{C}_{i+1}^{0} \oplus \mathcal{C}_{i}^{1} \oplus \cdots \oplus \mathcal{C}_{i-n+1}^{n} & \longrightarrow \mathcal{C}_{i}^{0} \oplus \mathcal{C}_{i-1}^{1} \oplus \cdots \oplus \mathcal{C}_{i-n}^{n} \\
\left(x_{0}, x_{1}, \ldots, x_{n}\right) & \longmapsto\left(h_{i}^{0}\left(x_{1}\right), h_{i-1}^{1}\left(x_{2}\right), \ldots, h_{i-n+1}^{n-1}\left(x_{n}\right), 0\right)
\end{aligned}
$$

and $\bar{H}=\left(\bar{H}_{i}\right)_{i \in \mathbb{Z}}$ defined analogously on $(\overline{\mathcal{C}}, \bar{d}, \bar{\partial})$. Then if $\bar{D}$ and $D$ are the differentials of Cone $\left(\bar{\partial}^{0}, \ldots, \bar{\partial}^{n-1}\right)$ and $\operatorname{Cone}\left(\partial^{0}, \ldots, \partial^{n-1}\right)$, we have

$$
F G=\mathrm{id}+H D+D H \quad \text { and } \quad G F=\mathrm{id}+\bar{H} \bar{D}+\bar{D} \bar{H} .
$$




\subsection{Fundamental splitting lemmas}

Let $(\Gamma, \epsilon)$ be an OMS-divide or a cuspidal divide. Let $p$ be a double point or a vertical tangent point of $\Gamma$. Let $\Gamma^{0}$ and $\Gamma^{1}$ be the cuspidal divides obtained from $\Gamma$ by applying $\Theta_{0}$ and $\Theta_{1}$ at $p$ respectively. Then each enhanced state of $\Gamma$ can be identified with either an enhanced state of $\Gamma^{0}$ or $\Gamma^{1}$ ie

$$
\widetilde{\operatorname{St}}(\Gamma) \stackrel{1-1}{\simeq} \widetilde{\operatorname{St}}\left(\Gamma^{0}\right) \sqcup \widetilde{\operatorname{St}}\left(\Gamma^{1}\right) .
$$

Consequently, $\llbracket \Gamma^{0} \rrbracket$ and $\llbracket \Gamma^{1} \rrbracket$ can be seen as sub-vector spaces of $\llbracket \Gamma \rrbracket$ up to translations of the grading $i$ and the degree $j$. More precisely, we have:

Lemma 3.13 Let $d$ be the differential of $\llbracket \Gamma \rrbracket$.

(1) If $p$ is a positive double point then $d$ induces the differentials $d^{0}$ and $d^{1}$ of $\llbracket \Gamma^{0} \rrbracket\{2\}$ and $\llbracket \Gamma^{1} \rrbracket\{4\}$ and a morphism

$$
\llbracket \Gamma^{0} \rrbracket\{2\} \stackrel{d^{\bullet}}{\longrightarrow} \llbracket \Gamma^{1} \rrbracket\{4\}
$$

such that $\llbracket \Gamma \rrbracket=\operatorname{Cone}\left(d^{\bullet}\right)$.

(2) If $p$ is a negative double point then $d$ induces the differentials $d^{0}$ and $d^{1}$ of $\llbracket \Gamma^{0} \rrbracket\{-2\}$ and $\llbracket \Gamma^{1} \rrbracket\{-4\}$ and a morphism

$$
\llbracket \Gamma^{1} \rrbracket\{-4\} \stackrel{d^{\bullet}}{\longrightarrow} \llbracket \Gamma^{0} \rrbracket\{-2\}
$$

such that $\llbracket \Gamma \rrbracket=\operatorname{Cone}\left(d^{\bullet}\right)[-1]$.

(3) If $p$ is vertical tangent point, then $d$ induces the differentials $d^{0}$ and $d^{1}$ of $\llbracket \Gamma^{0} \rrbracket\{1\}$ and $\llbracket \Gamma^{1} \rrbracket\{2\}$ and a morphism

$$
\llbracket \Gamma^{0} \rrbracket\{1\} \stackrel{d^{\bullet}}{\longrightarrow} \llbracket \Gamma^{1} \rrbracket\{2\}
$$

such that $\llbracket \Gamma \rrbracket=\operatorname{Cone}\left(d^{\bullet}\right)$.

Proof Suppose that $p$ is a positive double point of $\Gamma$. Then $\Gamma^{0}$ and $\Gamma^{1}$ have one positive double point less than $\Gamma$ so that the writhes of $\Gamma, \Gamma^{0}$ and $\Gamma^{1}$ are related by

$$
w(\Gamma)=w\left(\Gamma^{0}\right)+2=w\left(\Gamma^{1}\right)+2
$$

Let $\widetilde{S}$ be an enhanced state of $\widetilde{\operatorname{St}}_{i}(\Gamma)$ with degree $j=j(\widetilde{S})$. If $\widetilde{S}$ is obtained from $\Gamma$ using $\Theta_{0}$ (resp. $\left.\Theta_{1}\right)$ at $\underset{\sim}{p}$, then $\widetilde{S}$ can be seen as an enhanced state of $\widetilde{S t}_{i}\left(\Gamma^{0}\right)$ with degree $j-2$ (resp. of $\widetilde{\mathrm{St}}_{i-1}\left(\Gamma^{1}\right)$ with degree $\left.j-4\right)$. Besides, if $\widetilde{S}_{0} \in \widetilde{\mathrm{St}}_{i}(\Gamma)$ and $\widetilde{S}_{1} \in \widetilde{\mathrm{St}}_{i+1}(\Gamma)$ are adjacent enhanced states of degrees $j$ then it involves three cases: 
- Either $\widetilde{S}_{0} \in \widetilde{\operatorname{St}}_{i}\left(\Gamma^{0}\right)$ and $\widetilde{S}_{1} \in \widetilde{\operatorname{St}}_{i+1}\left(\Gamma^{0}\right)$ are adjacent enhanced states of $\Gamma^{0}$ with degrees $j-2$, so the differential $d^{0}$ of $\llbracket \Gamma^{0} \rrbracket\{2\}$ coincides with the restriction of $d$ to $\llbracket \Gamma^{0} \rrbracket\{2\}$; or

- $\widetilde{S}_{0} \in \widetilde{\mathrm{St}}_{i-1}\left(\Gamma^{1}\right)$ and $\widetilde{S}_{1} \in \widetilde{\mathrm{St}}_{i}\left(\Gamma^{1}\right)$ are adjacent enhanced states of $\Gamma^{1}$ with degrees $j-4$, so the differential $d^{1}$ of $\llbracket \Gamma^{1} \rrbracket\{4\}[1]$ coincides with the restriction of $d$ to $\llbracket \Gamma \rrbracket\{4\}[1]$; or

- $\widetilde{S}_{0} \in \widetilde{\mathrm{St}}_{i}\left(\Gamma^{0}\right)$ with degree $j-2$ and $\widetilde{S}_{1} \in \widetilde{\mathrm{St}}_{i}\left(\Gamma^{1}\right)$ with degree $j-4$, then $d$ induces a map $d^{\bullet}: \llbracket \Gamma^{0} \rrbracket\{2\} \rightarrow \llbracket \Gamma^{1} \rrbracket\{4\}$ which is a morphism of complexes since from the proof of Proposition $3.3 d d=0$ implies $d^{\bullet} d^{0}=d^{1} d^{\bullet}$.

Hence $\llbracket \Gamma \rrbracket=\operatorname{Cone}\left(d^{\bullet}\right)$. Similar arguments hold for the two other cases.

More generally, consider $k=k_{+}+k_{-}+k_{0}$ double vertical tangent points $p_{1}, \ldots, p_{k}$ such that the $k_{+}$first ones are positive double points, the next $k_{-}$ones negative double points and the last $k_{0}$ ones vertical tangent points. For each words $[a]=a_{1} a_{2} \cdots a_{k_{+}}$, $a_{i} \in\{0,1\},[b]=b_{1} b_{2} \cdots b_{k_{-}}, b_{i} \in\{0,1\},[c]=c_{1} c_{2} \cdots c_{k_{0}}, c_{i} \in\{0,1\}$, let $[a][b][c]$ be the word obtained by concatenation of $[a],[b],[c]$ and denote by $\left(\Gamma^{[a][b][c]}, \epsilon^{[a][b][c]}\right)$ the cuspidal divide obtained from $(\Gamma, \epsilon)$ by performing:

$$
\begin{cases}\Theta_{a_{i}} & \text { splitting at } p_{i} \text { for } 1 \leq i \leq k_{+} \\ \Theta_{b_{i}} & \text { splitting at } p_{i} \text { for } k_{+}<i \leq k_{+}+k_{-} \\ \Theta_{c_{i}} & \text { splitting at } p_{i} \text { for } k_{+}+k_{-}<i \leq k=k_{+}+k_{-}+k_{0} .\end{cases}
$$

Let $1_{[a]}, 1_{[b]}$ and $1_{[c]}$ be the numbers of occurrences of 1 in $[a],[b]$ and $[c]$ and $\operatorname{gr}([a][b][c])=1_{[a]}-1_{[b]}+1_{[c]}$. By restriction, the differential $d$ of $\llbracket \Gamma \rrbracket$ coincides with the differential $d^{[a][b][c]}$ of $\llbracket \Gamma^{[a][b][c]} \rrbracket$. By iterating Lemma 3.13, using same arguments, just following the incidence relations, we have:

Lemma 3.14 For each $\ell,-k_{-} \leq \ell \leq k_{+}+k_{0}$, we can identify the complex

$$
\left(\mathcal{C}^{\ell}, D^{\ell}\right)=\bigoplus_{\operatorname{gr}([a][b][c])=\ell} \llbracket \Gamma^{[a][b][c]} \rrbracket\left\{2\left(1_{[a]}-1_{[b]}+k_{+}-k_{-}\right)+1_{[c]}+k_{0}\right\}
$$

with a subquotient-complex of $\llbracket \Gamma \rrbracket$, with differential

$$
D^{\ell}=\bigoplus_{\operatorname{gr}([a][b][c])=\ell} d^{[a][b][c]} .
$$


The differential $d$ induces a structure of double complex

$$
\begin{aligned}
&\left(\mathcal{C}^{-k_{-}}, D^{-k_{-}}\right) \stackrel{\Delta^{-k_{-}}}{\longrightarrow}\left(\mathcal{C}^{-k_{-}+1}, D^{-k_{-}+1}\right) \stackrel{\Delta^{-k_{-}+1}}{\longrightarrow} \cdots \\
& \stackrel{\Delta^{k_{+}+k_{0}-1}}{\longrightarrow}\left(\mathcal{C}^{k_{+}+k_{0}}, D^{k_{+}+k_{0}}\right)
\end{aligned}
$$

such that $\llbracket \Gamma \rrbracket=$ Cone $\left(\Delta^{-k_{-}}, \ldots, \Delta^{k_{+}+k_{0}-1}\right)\left[-k_{-}\right]$.

In the sequel, such a double complex will be called a splitting diagram of $\llbracket \Gamma \rrbracket$.

\subsection{Khovanov homology of OMS-divides}

Definition 3.15 We call Khovanov homology $\mathcal{H}(\Gamma)$ of an OMS-divide (or a cuspidal divide) $\Gamma=(\Gamma, \epsilon)$ the homology of the complex $\llbracket \Gamma \rrbracket=\left(\llbracket \Gamma \rrbracket_{i}\right)_{i \in \mathbb{Z}}$ :

$$
\begin{gathered}
\mathcal{H}(\Gamma)=\left(\mathcal{H}_{i}(\Gamma)\right)_{i \in \mathbb{Z}}, \quad \mathcal{H}_{i}(\Gamma)=\bigoplus_{j \in \mathbb{Z}} \mathcal{H}_{i, j}(\Gamma), \\
\mathcal{H}_{i, j}(\Gamma)=\operatorname{Ker} d_{i, j} / \operatorname{Im} d_{i-1, j}
\end{gathered}
$$

Proposition 3.16 If $\Gamma=(\Gamma, \epsilon)$ is an OMS-divide, then the polynomial $W_{\Gamma}$ and the graded Euler characteristics of $\mathcal{H}(\Gamma)$ are related by

$$
W_{\Gamma}\left(t^{2}\right)=\frac{t}{1+t^{2}} \chi_{\mathrm{gr}}(\mathcal{H}(\Gamma))=\frac{t}{1+t^{2}} \sum_{i \in \mathbb{Z}}(-1)^{i} \operatorname{dim}_{\mathrm{gr}} \mathcal{H}_{i}(\Gamma)
$$

where the graded dimension is $\operatorname{dim}_{\mathrm{gr}} \mathcal{H}_{i}(\Gamma)=\sum_{j \in \mathbb{Z}} t^{j} \operatorname{dim}_{\mathbb{Z}_{2}} \mathcal{H}_{i, j}(\Gamma)$.

Proof Immediate from formula (2-5).

We can now formulate our main theorem:

Theorem 3.17 Khovanov homology of OMS-divides is invariant under $\mathcal{M}$-equivalence.

Combined with Theorem 1.2, we obtain:

Corollary 3.18 Khovanov homology of OMS-divides is an invariant for strong equivalence of strongly invertible links.

Section 4 is devoted to the proof of this theorem. Notice that from Proposition 3.16, Theorem 3.17 is a refinement of Proposition 2.3. 


\subsection{Examples}

(1) Figure 9 shows a divide for the link $3_{1}$ and its splitting diagram.
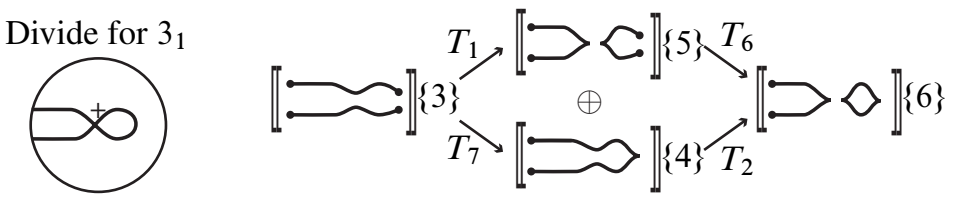

Figure 9

The associated complex and homology entries are:

$$
(\mathcal{A} \otimes \mathcal{A})\{3\} \stackrel{d^{0}}{\rightarrow}(\mathcal{A} \otimes \mathcal{A})\{5\} \oplus \mathcal{A}\{4\} \stackrel{d^{1}}{\rightarrow}(\mathcal{A} \otimes \mathcal{B})\{6\}
$$

\begin{tabular}{|c|c|c|c|c|c|}
\hline$i \backslash j$ & 1 & 3 & 5 & 7 & 9 \\
\hline 0 & $\mathbb{Z}_{2}$ & $\mathbb{Z}_{2}$ & & & \\
\hline 1 & & & $\mathbb{Z}_{2}$ & $\mathbb{Z}_{2}$ & \\
\hline 2 & & & & $\mathbb{Z}_{2}$ & $\mathbb{Z}_{2}$ \\
\hline
\end{tabular}

(2) Figure 10 shows a divide for the link $4_{1}$ and its splitting diagram.

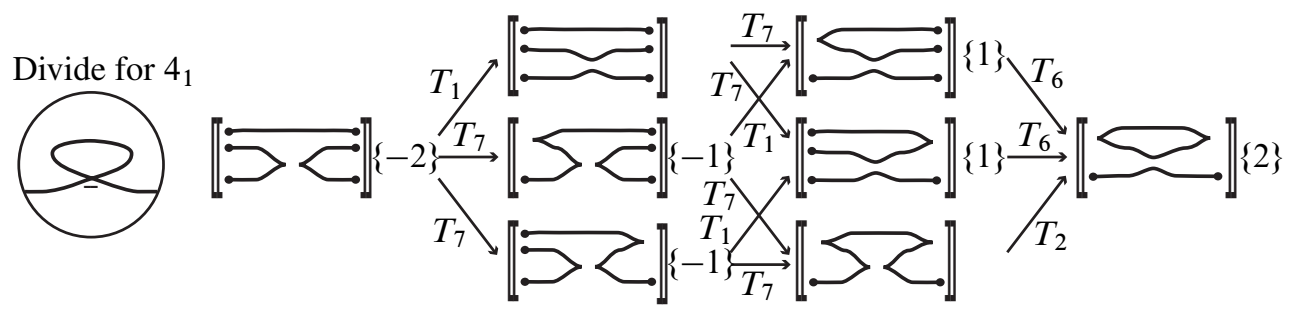

Figure 10

The associated complex and homology entries are:

$\left(\mathcal{A}^{\otimes 3}\right)\{-2\} \stackrel{d^{-1}}{\rightarrow}\left(\mathcal{A}^{\otimes 3}\right) \oplus\left(\mathcal{A}^{\otimes 2} \oplus \mathcal{A}^{\otimes 2}\right)\{-1\} \stackrel{d^{0}}{\rightarrow}\left(\mathcal{A}^{\otimes 2} \oplus \mathcal{A}^{\otimes 2}\right)\{1\} \oplus \mathcal{A} \stackrel{d^{1}}{\rightarrow}(\mathcal{A} \otimes \mathcal{B})\{2\}$

\begin{tabular}{|c|c|c|c|c|c|c|}
\hline$i \backslash j$ & -5 & -3 & -1 & 1 & 3 & 5 \\
\hline-1 & $\mathbb{Z}_{2}$ & $\mathbb{Z}_{2}$ & & & & \\
\hline 0 & & $\mathbb{Z}_{2}$ & $\left(\mathbb{Z}_{2}\right)^{2}$ & $\mathbb{Z}_{2}$ & & \\
\hline 1 & & & & $\mathbb{Z}_{2}$ & $\mathbb{Z}_{2}$ & \\
\hline 2 & & & & & $\mathbb{Z}_{2}$ & $\mathbb{Z}_{2}$ \\
\hline
\end{tabular}




\section{Invariance under $\mathcal{M}$-equivalence}

\subsection{Invariance under type I moves}

Let $\Gamma$ and $\widetilde{\Gamma}$ be OMS-divides which differ only by a type I move (see Figure 11).

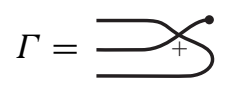

$$
\tilde{\Gamma}=\longrightarrow
$$

Figure 11: Type I move

Proposition 4.1 The complexes $\llbracket \Gamma \rrbracket$ and $\llbracket \widetilde{\Gamma} \rrbracket$ have the same homology.

Let's denote by $\Gamma^{s t}\left(\right.$ resp. $\left.\tilde{\Gamma}^{s t}\right), s, t \in\{0,1\}$ the cuspidal divides obtained by performing $\Theta_{s}, \Theta_{t}$ splittings respectively at the + double point and the vertical tangent point of $\Gamma$ (resp. of $\tilde{\Gamma}$ ) in Figure 11, without changing any other singular point of these divides. From Lemma 3.14, we have splitting diagrams given in Figure 12.

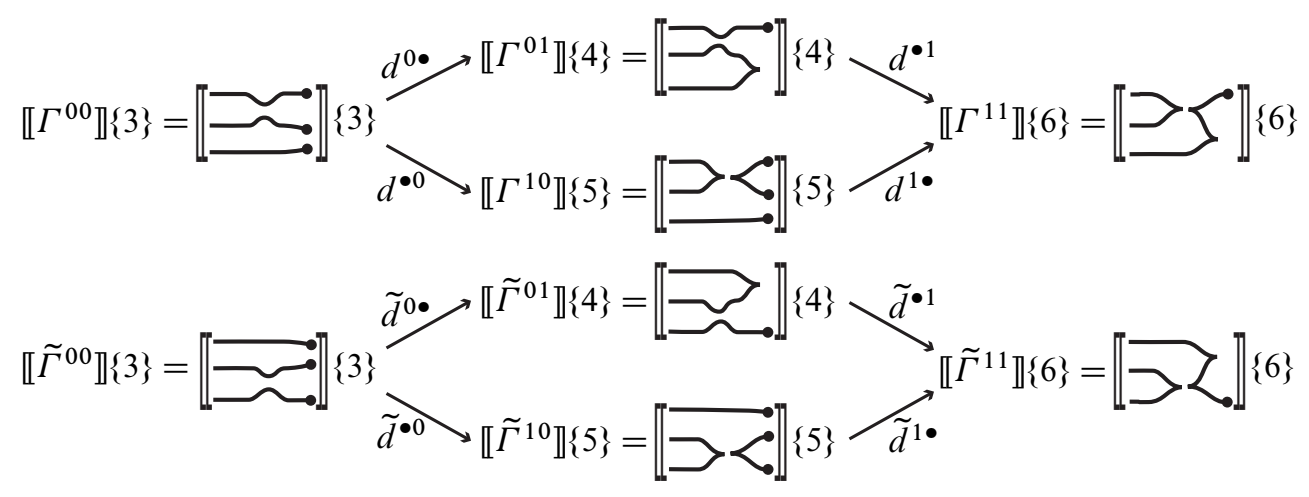

Figure 12: Splitting diagram for type I move

Notice that $\llbracket \Gamma^{00} \rrbracket=\llbracket \tilde{\Gamma}^{00} \rrbracket$. In other words we have:

Lemma 4.2 Let's denote

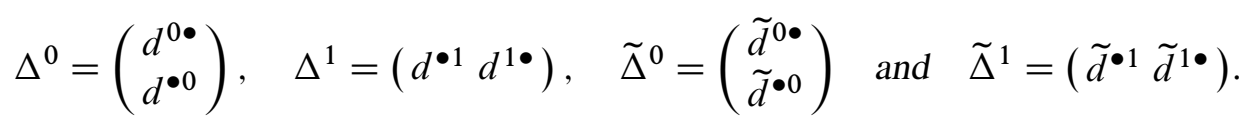


Then $\llbracket \Gamma \rrbracket=\operatorname{Cone}\left(\Delta^{0}, \Delta^{1}\right)$ and $\llbracket \tilde{\Gamma} \rrbracket=\operatorname{Cone}\left(\widetilde{\Delta}^{0}, \widetilde{\Delta}^{1}\right)$ :

$$
\begin{aligned}
& \llbracket \Gamma^{00} \rrbracket\{3\} \stackrel{\Delta^{0}}{\longrightarrow} \llbracket \Gamma^{01} \rrbracket\{4\} \oplus \llbracket \Gamma^{10} \rrbracket\{5\} \stackrel{\Delta^{1}}{\longrightarrow} \llbracket \Gamma^{11} \rrbracket\{6\} \\
& \llbracket \tilde{\Gamma}^{00} \rrbracket\{3\} \stackrel{\widetilde{\Delta}^{0}}{\longrightarrow} \llbracket \tilde{\Gamma}^{01} \rrbracket\{4\} \oplus \llbracket \tilde{\Gamma}^{10} \rrbracket\{5\} \stackrel{\tilde{\Delta}^{1}}{\longrightarrow} \llbracket \tilde{\Gamma}^{11} \rrbracket\{6\}
\end{aligned}
$$

Let's consider the "creation and destruction" morphisms (see Remark 3.6)

$$
\begin{array}{llll}
\llbracket \Gamma^{11} \rrbracket\{6\} \stackrel{\eta_{1}}{\longrightarrow} \llbracket \Gamma^{10} \rrbracket\{5\} & \llbracket \Gamma^{01} \rrbracket\{4\} \stackrel{\bar{\eta}_{1}}{\longrightarrow} \llbracket \tilde{\Gamma}^{10} \rrbracket\{5\} & \llbracket \tilde{\Gamma}^{10} \rrbracket\{5\} \stackrel{\widetilde{\varepsilon}_{1}}{\longrightarrow} \llbracket \Gamma^{01} \rrbracket\{4\} \\
\llbracket \tilde{\Gamma}^{11} \rrbracket\{6\} \stackrel{\tilde{\eta}_{1}}{\longrightarrow} \llbracket \tilde{\Gamma}^{10} \rrbracket\{5\} & \llbracket \tilde{\Gamma}^{01} \rrbracket\{4\} \stackrel{\tilde{\bar{\eta}}_{1}}{\longrightarrow} \llbracket \Gamma^{10} \rrbracket\{5\} & \llbracket \Gamma^{10} \rrbracket\{5\} \stackrel{\varepsilon_{1}}{\longrightarrow} \llbracket \tilde{\Gamma}^{01} \rrbracket\{4\}
\end{array}
$$

defined by Figure 13.

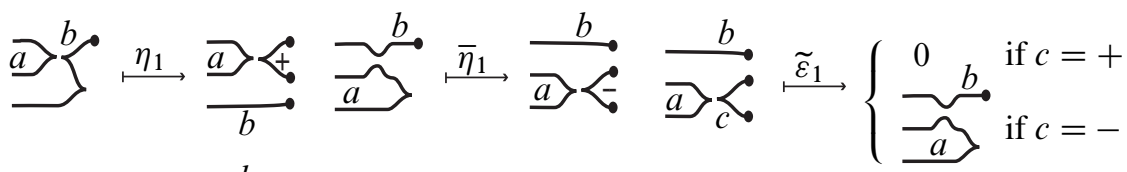

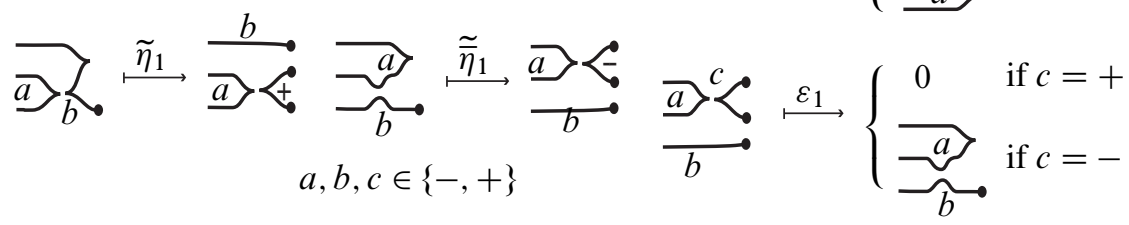

Figure 13

Lemma 4.3 The two sequences

$$
\begin{aligned}
& 0 \longrightarrow \llbracket \Gamma^{11} \rrbracket\{6\} \stackrel{\eta_{1}}{\longrightarrow} \llbracket \Gamma^{10} \rrbracket\{5\} \stackrel{\varepsilon_{1}}{\longrightarrow} \llbracket \widetilde{\Gamma}^{01} \rrbracket\{4\} \longrightarrow 0 \\
& 0 \longrightarrow \llbracket \widetilde{\Gamma}^{11} \rrbracket\{6\} \stackrel{\tilde{\eta}_{1}}{\longrightarrow} \llbracket \widetilde{\Gamma}^{10} \rrbracket\{5\} \stackrel{\widetilde{\varepsilon}_{1}}{\longrightarrow} \llbracket \Gamma^{01} \rrbracket\{4\} \longrightarrow 0
\end{aligned}
$$

are exact and $d^{1 \bullet}, \widetilde{\bar{\eta}}_{1}, \tilde{d}^{1 \bullet}$ and $\bar{\eta}_{1}$ are respectively splitting morphisms of $\eta_{1}, \varepsilon_{1}, \tilde{\eta}_{1}$ and $\widetilde{\varepsilon}_{1}$ :

$$
\begin{array}{lll}
d^{1 \bullet} \eta_{1}=\mathrm{id}, & \varepsilon_{1} \tilde{\bar{\eta}}_{1}=\mathrm{id}, & \eta_{1} d^{1 \bullet}+\tilde{\bar{\eta}}_{1} \varepsilon_{1}=\mathrm{id}+\eta_{1} d^{1 \bullet} \tilde{\bar{\eta}}_{1} \varepsilon_{1} \\
\tilde{d}^{1 \bullet} \tilde{\eta}_{1}=\mathrm{id}, & \widetilde{\varepsilon}_{1} \bar{\eta}_{1}=\mathrm{id}, & \tilde{\eta}_{1} \tilde{d}^{1 \bullet}+\bar{\eta}_{1} \tilde{\varepsilon}_{1}=\mathrm{id}+\tilde{\eta}_{1} \tilde{d}^{1 \bullet} \bar{\eta}_{1} \widetilde{\varepsilon}_{1}
\end{array}
$$

Moreover

$$
\varepsilon_{1} d^{\bullet 0}=\tilde{d}^{0 \bullet} \quad \text { and } \quad \tilde{\varepsilon}_{1} \tilde{d}^{\bullet 0}=d^{0 \bullet} .
$$


Proof The morphisms $d^{1 \bullet}$ and $\tilde{d}^{1 \bullet}$ correspond to $T_{7}, d^{\bullet 0}$ and $\tilde{d}^{\bullet 0}$ to $T_{1}$ or $T_{2}, d^{0 \bullet}$ and $\tilde{d}^{0 \bullet}$ to $T_{6}$ or $T_{4}$ (see (3-16)). Then the result is an consequence of the comments and the definitions made in Remark 3.6 (see also Figure 6 and Figure 8). Indeed, with the notation of this remark, relations (4-3) correspond to

$$
\begin{aligned}
& T_{7} \circ\left(A \otimes \eta_{1}\right)=\mathrm{id} \quad \varepsilon_{1} \circ \bar{\eta}_{1}=\mathrm{id} \\
& \left(A \otimes \eta_{1}\right) \circ T_{7}+\left(A \otimes\left(\bar{\eta}_{1} \varepsilon_{1}\right)\right)=\mathrm{id}+\left(A \otimes \eta_{1}\right) \circ T_{7} \circ\left(A \otimes\left(\bar{\eta}_{1} \varepsilon_{1}\right)\right)
\end{aligned}
$$

and relations (4-4) to $\left(\varepsilon_{1} \otimes A\right) \circ T_{1}=T_{6}$ or $\left(\varepsilon_{1} \otimes B\right) \circ T_{2}=T_{4}$.

Proof of Proposition 4.1 Consider the diagram

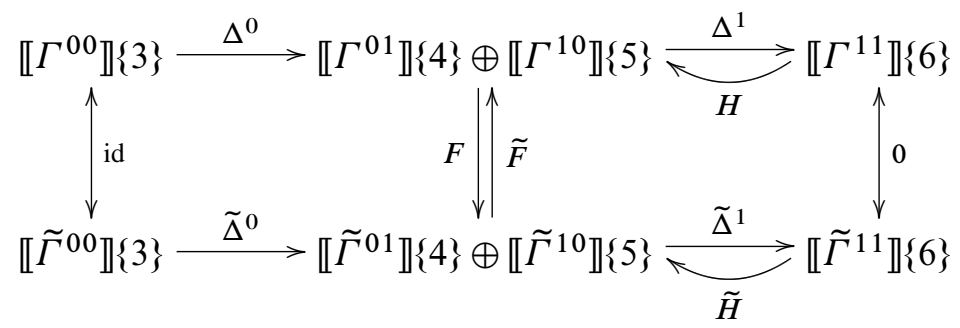

where $\quad H=\left(\begin{array}{c}0 \\ \eta_{1}\end{array}\right)$

$$
\widetilde{H}=\left(\begin{array}{c}
0 \\
\tilde{\eta}_{1}
\end{array}\right)
$$

$$
F=\left(\begin{array}{cc}
0 & \varepsilon_{1} \\
\bar{\eta}_{1}+\tilde{\eta}_{1} \tilde{d}^{1 \bullet} \bar{\eta}_{1} & \tilde{\eta}_{1} \tilde{d}^{\bullet 1} \varepsilon_{1}
\end{array}\right) \quad \widetilde{F}=\left(\begin{array}{cc}
0 & \widetilde{\varepsilon}_{1} \\
\tilde{\bar{\eta}}_{1}+\eta_{1} d^{1 \bullet} \tilde{\bar{\eta}}_{1} & \eta_{1} d^{\bullet 1} \widetilde{\varepsilon}_{1}
\end{array}\right) .
$$

From Lemmas 4.2 and 4.3, we have

$$
\begin{array}{llll}
F \Delta^{0}=\tilde{\Delta}^{0} & \tilde{\Delta}^{1} F=0 & \widetilde{F} F=\mathrm{id}+H \Delta^{1} & \Delta^{1} H=\mathrm{id} \\
\tilde{F} \widetilde{\Delta}^{0}=\Delta^{0} & \Delta^{1} \tilde{F}=0 & F \tilde{F}=\mathrm{id}+\tilde{H} \widetilde{\Delta}^{1} & \widetilde{\Delta}^{1} \tilde{H}=\mathrm{id} .
\end{array}
$$

Hence vertical arrows define a homotopy equivalence. From Proposition 3.12, $\llbracket \Gamma \rrbracket=$ $\operatorname{Cone}\left(\Delta^{0}, \Delta^{1}\right)$ and $\llbracket \tilde{\Gamma} \rrbracket=\operatorname{Cone}\left(\widetilde{\Delta}^{0}, \widetilde{\Delta}^{1}\right)$ have the same homology.

\subsection{Invariance under type II move}

Let $\Gamma$ and $\Gamma_{0}$ be OMS-divides which differ only by a type II move (see Figure 14).
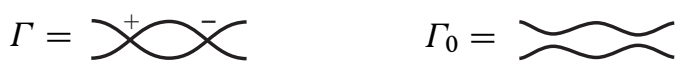

Figure 14: Type II move 
Proposition 4.4 The complexes $\llbracket \Gamma \rrbracket$ and $\llbracket \Gamma_{0} \rrbracket$ have the same homology.

Let $\Gamma^{s t}, s, t \in\{0,1\}$ be the cuspidal divides obtained by performing $\Theta_{s}$ and $\Theta_{t}$ splittings respectively at the + and the - double points of $\Gamma$ in Figure 14 without changing any other singular point. From Lemma 3.14 we have a splitting diagram given by Figure 15 .

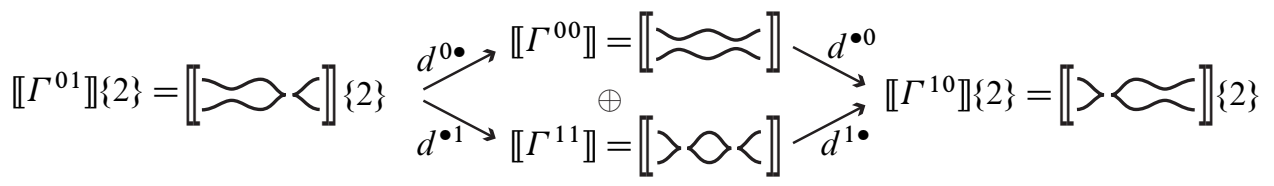

Figure 15: Splitting diagram for type II move

We remark that $\llbracket \Gamma^{00} \rrbracket=\llbracket \Gamma_{0} \rrbracket$ and we have the following lemma:

Lemma $4.5 \llbracket \Gamma \rrbracket=\operatorname{Cone}\left(\Delta^{0}, \Delta^{1}\right)[-1]$ where

$\llbracket \Gamma^{01} \rrbracket\{-2\} \stackrel{\Delta^{0}}{\longrightarrow} \llbracket \Gamma^{00} \rrbracket \oplus \llbracket \Gamma^{11} \rrbracket \stackrel{\Delta^{1}}{\longrightarrow} \llbracket \Gamma^{10} \rrbracket\{2\} \quad \Delta^{0}=\left(\begin{array}{l}d^{0 \bullet} \\ d^{\bullet 1}\end{array}\right) \quad \Delta^{1}=\left(d^{\bullet 0} d^{1 \bullet}\right)$.

Let's consider the "destruction and creation" morphisms of complexes (see Remark 3.6) defined by Figure 16.

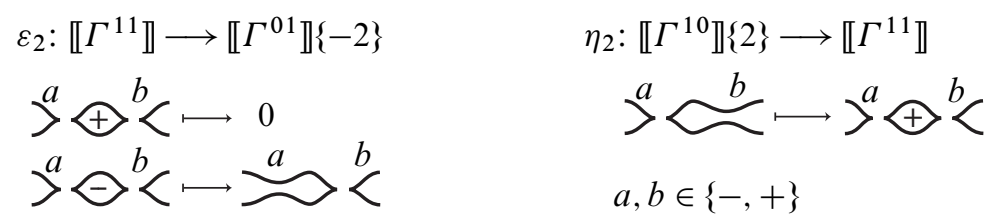

Figure 16

Lemma 4.6 The sequence

$$
0 \longrightarrow \llbracket \Gamma^{10} \rrbracket\{2\} \stackrel{\eta_{2}}{\longrightarrow} \llbracket \Gamma^{11} \rrbracket \stackrel{\varepsilon_{2}}{\longrightarrow} \llbracket \Gamma^{01} \rrbracket\{-2\} \longrightarrow 0
$$

is exact and $d^{1 \bullet}, d^{\bullet 1}$ are respectively splitting morphisms of $\eta_{2}, \varepsilon_{2}$ :

$$
\varepsilon_{2} d^{\bullet 1}=\mathrm{id}, \quad d^{1 \bullet} \eta_{2}=\mathrm{id}, \quad d^{\bullet 1} \varepsilon_{2}+\eta_{2} d^{1 \bullet}=\mathrm{id}+\eta_{2} d^{1 \bullet} d^{\bullet 1} \varepsilon_{2} .
$$

Proof The morphism $d^{\bullet 1}$ corresponds to $T_{2}$ or $T_{4}$ and the morphism $d^{1 \bullet}$ to $T_{3}$ or $T_{5}$ in (3-16). The lemma is a direct consequence of the comments and the definitions made in Remark 3.6 (see also Figure 6 and Figure 7). 
Proof of Proposition 4.4 Consider the diagram

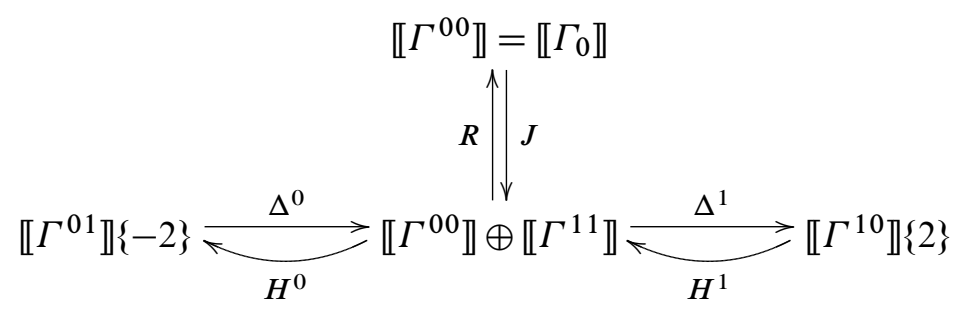

where

$$
\begin{aligned}
J & =\left(\begin{array}{c}
\mathrm{id} \\
\eta_{2} d^{\bullet 0}
\end{array}\right) & R & =\left(\mathrm{id} d^{0 \bullet} \varepsilon_{2}\right) \\
H^{0} & =\left(\begin{array}{ll}
0 & \varepsilon_{2}
\end{array}\right) & H^{1} & =\left(\begin{array}{c}
0 \\
\eta_{2}
\end{array}\right)
\end{aligned}
$$

From the previous two lemmas, $\llbracket \Gamma_{0} \rrbracket$ is a strong deformation retract of $\llbracket \Gamma \rrbracket=$ $\operatorname{Cone}\left(\Delta^{0}, \Delta^{1}\right)[-1]$ with retraction $R$, inclusion $J$, and homotopy $\left(H^{0}, H^{1}\right)$ :

$R \Delta^{0}=0, \Delta^{1} J=0, H^{0} \Delta^{0}=\mathrm{id}, R J=\mathrm{id}, J R=\mathrm{id}+\Delta^{0} H^{0}+H^{1} \Delta^{1}, \Delta^{1} H^{1}=\mathrm{id}$.

Hence from Proposition 3.12 they have the same homology.

\subsection{Invariance under type III move}

In this section, we only consider the case of move $\mathrm{III}_{+}$. The case of III- can be checked in a similar way: we have dual situations as is said in Remark 3.2 and in the proof of Proposition 3.5.

Let $\Gamma_{1}$ and $\Gamma_{2}$ be OMS-divides which differ only by a type III + move (see Figure 17).
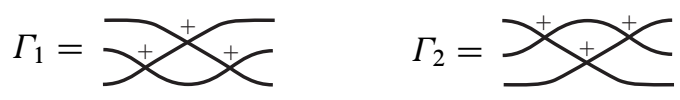

Figure 17: Type $\mathrm{III}_{+}$move

Proposition 4.7 The complexes $\llbracket \Gamma_{1} \rrbracket$ and $\llbracket \Gamma_{2} \rrbracket$ have the same homology.

Let $\Gamma_{1}^{s t u}, s, t, u \in\{0,1\}$ be the cuspidal divides obtained by performing $\Theta_{s}, \Theta_{t}$ and $\Theta_{u}$ splittings at the double points shown on the figure of $\Gamma_{1}$ (see Figure 17). From Lemma 3.14, we have the splitting diagram of $\llbracket \Gamma_{1} \rrbracket$ shown in Figure 18. 


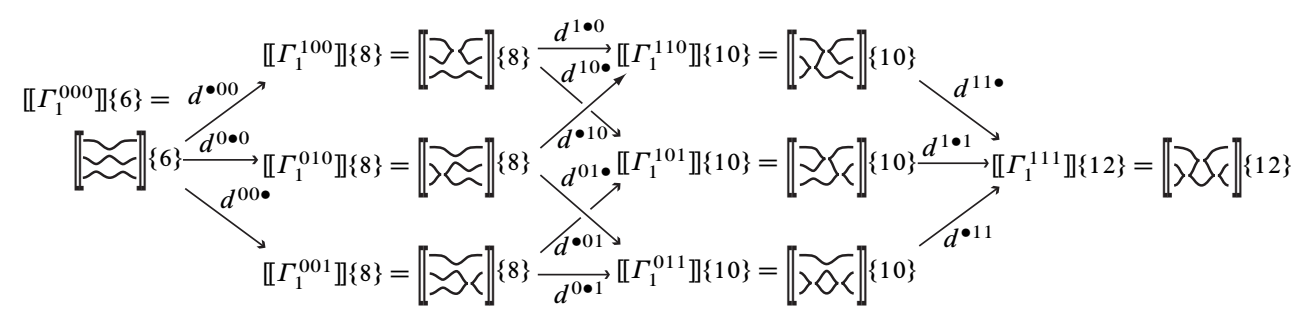

Figure 18: Splitting diagram for type $\mathrm{III}_{+}$move

Let's denote $\mathcal{C}^{0}=\llbracket \Gamma_{1}^{000} \rrbracket\{6\}, \mathcal{C}^{3}=\llbracket \Gamma_{1}^{111} \rrbracket\{12\}, \mathcal{C}^{1}=\widehat{\mathcal{C}}^{1} \oplus \check{\mathcal{C}}^{1}$ and $\mathcal{C}^{2}=\widehat{\mathcal{C}}^{2} \oplus \check{\mathcal{C}}^{2}$ where

$$
\begin{array}{llrl}
\widehat{\mathcal{C}}^{1} & =\llbracket \Gamma_{1}^{100} \rrbracket\{8\} \oplus \llbracket \Gamma_{1}^{010} \rrbracket\{8\} & \check{\mathcal{C}}^{1}=\llbracket \Gamma_{1}^{001} \rrbracket\{8\} \\
\widehat{\mathcal{C}}^{2}=\llbracket \Gamma_{1}^{110} \rrbracket\{10\} \oplus \llbracket \Gamma_{1}^{101} \rrbracket\{10\} & \check{\mathcal{C}}^{2}=\llbracket \Gamma_{1}^{011} \rrbracket\{10\} .
\end{array}
$$

Lemma 4.8 $\llbracket \Gamma_{1} \rrbracket=\operatorname{Cone}\left(\Delta^{0}, \Delta^{1}, \Delta^{2}\right)$ where

$$
\mathcal{C}^{0} \stackrel{\Delta^{0}}{\longrightarrow} \mathcal{C}^{1} \stackrel{\Delta^{1}}{\longrightarrow} \mathcal{C}^{2} \stackrel{\Delta^{2}}{\longrightarrow} \mathcal{C}^{3}
$$

and $\quad \Delta^{0}=\left(\begin{array}{l}d^{\bullet 00} \\ d^{0 \bullet 0} \\ \hdashline d^{00 \bullet}\end{array}\right) \quad \Delta^{1}=\left(\begin{array}{cc:c}d^{1 \bullet 0} & d^{\bullet 10} & 0 \\ d^{10 \bullet} & 0 & d^{\bullet 01} \\ \hdashline 0 & d^{01 \bullet} & d^{0 \bullet 1}\end{array}\right) \quad \Delta^{2}=\left(\begin{array}{ll}11 \bullet & d^{1 \bullet 1}\end{array} d^{\bullet 11}\right)$.

Now we can use the same arguments as in Section 4.2. The morphism $d^{0 \bullet 1}$ (corresponding to $T_{2}$ and $T_{4}$ ) is injective and $d^{\bullet 11}$ (corresponding to $T_{3}$ and $T_{5}$ ) is surjective. Let $\varepsilon_{2}: \breve{\mathcal{C}}^{2} \rightarrow \breve{\mathcal{C}}^{1}$ be the "destruction" morphism of complexes and $\eta_{2}: \mathcal{C}^{3} \rightarrow \breve{\mathcal{C}}^{2}$ be the "creation" morphism of complexes (see Figure 19).
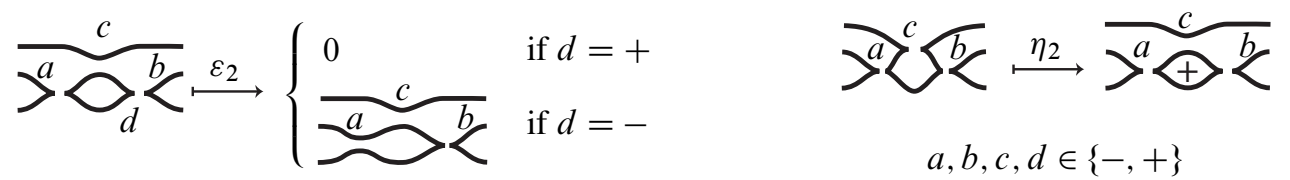
$a, b, c, d \in\{-,+\}$

Figure 19

Lemma 4.9 The sequence

$$
0 \longrightarrow \mathcal{C}^{3} \stackrel{\eta_{2}}{\longrightarrow} \check{\mathcal{C}}^{2} \stackrel{\varepsilon_{2}}{\longrightarrow} \check{\mathcal{C}}^{1} \longrightarrow 0
$$

is exact and $d^{\bullet 11}, d^{0 \bullet 1}$ are respectively splitting morphisms of $\eta_{2}$ and $\varepsilon_{2}$ :

$$
d^{\bullet 11} \eta_{2}=\mathrm{id}, \quad \varepsilon_{2} d^{0 \bullet 1}=\mathrm{id}, \quad d^{0 \bullet 1} \varepsilon_{2}+\eta_{2} d^{\bullet 11}=\mathrm{id}+\eta_{2} d^{\bullet 11} d^{0 \bullet 1} \varepsilon_{2} .
$$


Proof We have a similar situation as in Lemma 4.6: $d^{0 \bullet 1}$ corresponds to $T_{2}$ or $T_{4}$ and $d^{\bullet 11}$ corresponds to $T_{3}$ or $T_{5}$ in (3-16). The lemma is a direct consequence of the comments and the definitions made in Remark 3.6 (see also Figure 6).

Lemma 4.10 Let $\delta=d^{\bullet 01} \varepsilon_{2} d^{01 \bullet}: \llbracket \Gamma_{1}^{010} \rrbracket\{8\} \rightarrow \llbracket \Gamma_{1}^{101} \rrbracket\{10\}$.

The following sequence is a double-complex:

$\left(\mathcal{C}^{0}, d^{0}\right) \stackrel{\widehat{\Delta}^{0}}{\longrightarrow}\left(\widehat{\mathcal{C}}^{1}, \widehat{d}^{1}\right) \stackrel{\widehat{\Delta}^{1}}{\longrightarrow}\left(\widehat{\mathcal{C}}^{2}, \widehat{d}^{2}\right) \quad$ where $\quad \widehat{\Delta}^{0}=\left(\begin{array}{c}d^{\bullet 00} \\ d^{0 \bullet 0}\end{array}\right) \quad \widehat{\Delta}^{1}=\left(\begin{array}{cc}d^{1 \bullet 0} & d^{\bullet 10} \\ d^{10 \bullet} & \delta\end{array}\right)$.

Proof Since $\Delta^{1} \Delta^{0}=0$, we obtain from Lemma 4.9

$$
\begin{aligned}
& \widehat{\Delta}^{1} \widehat{\Delta}^{0}=\left(\begin{array}{c}
d^{1 \bullet 0} d^{\bullet 00}+d^{\bullet 10} d^{0 \bullet 0} \\
d^{10 \bullet} d^{\bullet 00}+\delta d^{0 \bullet 0}
\end{array}\right)=\left(\begin{array}{c}
0 \\
d^{10 \bullet} d^{\bullet 00}+d^{\bullet 01} \varepsilon_{2} d^{01 \bullet} d^{0 \bullet 0}
\end{array}\right), \\
& d^{10 \bullet} d^{\bullet 00}+d^{\bullet 01} \varepsilon_{2} d^{01 \bullet} d^{0 \bullet 0}=d^{\bullet 01} d^{00 \bullet}+d^{\bullet 01} \varepsilon_{2} d^{0 \bullet 1} d^{00 \bullet}=0 .
\end{aligned}
$$

Lemma 4.11 Let $\hat{\mathcal{C}}=\operatorname{Cone}\left(\widehat{\Delta}^{0}, \widehat{\Delta}^{1}\right)$. Then the complex $\widehat{\mathcal{C}}$ is a strong deformation retract of $\llbracket \Gamma_{1} \rrbracket$ and so they have the same homology.

Proof Consider the diagram

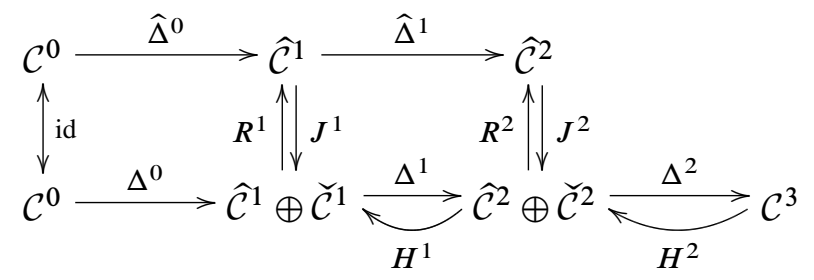

where $J^{1}=\left(\begin{array}{cc}\text { id } & 0 \\ 0 & \mathrm{id} \\ \hdashline 0 & \varepsilon_{2} d^{01} \bullet\end{array}\right) \quad R^{1}=\left(\begin{array}{cc:c}\mathrm{id} & 0 & 0 \\ 0 & \mathrm{id} & 0\end{array}\right) \quad H^{1}=\left(\begin{array}{cc:c}0 & 0 & 0 \\ 0 & 0 & 0 \\ \hdashline 0 & 0 & \varepsilon_{2}\end{array}\right)$

$$
J^{2}=\left(\begin{array}{cc}
\mathrm{id} & 0 \\
0 & \mathrm{id} \\
\hdashline \eta_{2} d^{11 \bullet} & \eta_{2} d^{1 \bullet 1}
\end{array}\right) \quad R^{2}=\left(\begin{array}{cc:c}
\mathrm{id} & 0 & 0 \\
0 & \mathrm{id} & d^{\bullet 01} \varepsilon_{2}
\end{array}\right) \quad H^{2}=\left(\begin{array}{c}
0 \\
0 \\
\hdashline \\
\eta_{2}
\end{array}\right) \text {. }
$$

From Lemma 4.9, we easily verify the relations:

$$
\begin{gathered}
J^{1} \widehat{\Delta}^{0}=\Delta^{0}, \quad J^{2} \widehat{\Delta}^{1}=\Delta^{1} J^{1}, \quad \Delta^{2} J^{2}=0, \quad R^{1} \Delta^{0}=\widehat{\Delta}^{0}, \quad R^{2} \Delta^{1}=\widehat{\Delta}^{1} R^{1}, \\
R^{1} J^{1}=\mathrm{id}, \quad R^{2} J^{2}=\mathrm{id}, J^{1} R^{1}=\mathrm{id}+H^{1} \Delta^{1}, \quad J^{2} R^{2}=\mathrm{id}+H^{2} \Delta^{2}+\Delta^{1} H^{1}, \\
0=\mathrm{id}+\Delta^{2} H^{2} .
\end{gathered}
$$


So downward arrows define an inclusion map, upward ones a retraction and $H_{1}, H_{2}$ homotopy maps of double complexes. We can apply Proposition 3.12: $\widehat{\mathcal{C}}$ is a deformation retract of $\llbracket \Gamma_{1} \rrbracket$ so they have the same homology.

Proof of Proposition 4.7 Up to isotopy, the drawn part of $\Gamma_{1}^{000}$ on Figure 18 is symmetric with respect to horizontal direction. Also the drawn part of $\Gamma_{1}^{100}$ is symmetric to the one of $\Gamma_{1}^{010}$ as well as the drawn part of $\Gamma_{1}^{110}$ is symmetric to the one of $\Gamma_{1}^{101}$. The morphisms $d^{\bullet 00}$ and $d^{0 \bullet 0}$ (resp. $d^{10 \bullet}$ and $d^{\bullet 10}$ ) clearly play symmetric roles. Besides, the morphisms $\delta: \llbracket \Gamma_{1}^{010} \rrbracket\{8\} \rightarrow \llbracket \Gamma_{1}^{101} \rrbracket\{10\}$ and $d^{1 \bullet 0}: \llbracket \Gamma_{1}^{100} \rrbracket\{8\} \rightarrow \llbracket \Gamma_{1}^{110} \rrbracket\{10\}$ also play symmetric roles. Since the drawn parts of $\Gamma_{1}$ and $\Gamma_{2}$ in Figure 17 also are symmetric with respect to horizontal direction, we deduce that the complexes $\llbracket \Gamma_{1} \rrbracket$ and $\llbracket \Gamma_{2} \rrbracket$ both have the same homology as the complex $\widehat{\mathcal{C}}$.

\subsection{Invariance under type IV moves}

Let $\Gamma_{1}$ and $\Gamma_{2}$ be OMS-divides which differ only by a type IV move (see Figure 20).
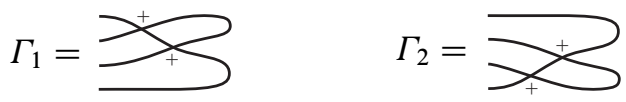

Figure 20: Type IV move

Proposition 4.12 The complexes $\llbracket \Gamma_{1} \rrbracket$ and $\llbracket \Gamma_{2} \rrbracket$ have the same homology.

Before proving this proposition, we first introduce the following intermediate result.

Lemma 4.13 Let $\Gamma$ and $\widetilde{\Gamma}$ be two cuspidal divides which differ only in the way depicted in Figure 21 (or the symmetric situation with respect to horizontal direction).

$$
\Gamma=\lessgtr \quad \tilde{\Gamma}=\supset
$$

Figure 21

Then $\llbracket \tilde{\Gamma} \rrbracket\{6\}[1]$ is a strong deformation retract of $\llbracket \Gamma \rrbracket$.

Proof With the same arguments as in the previous sections, using Lemma 3.14 we have a splitting diagram of $\llbracket \Gamma \rrbracket$ as in Figure 22. 


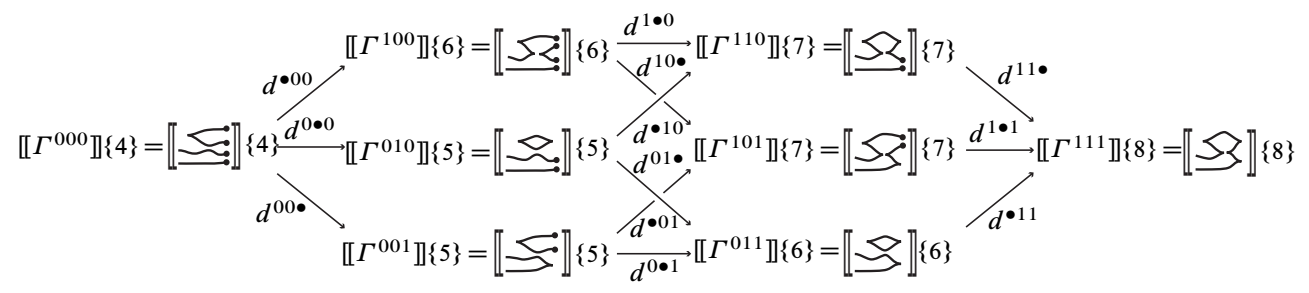

Figure 22: Splitting diagram of $\llbracket \Gamma \rrbracket$

Let's denote:

$$
\begin{array}{rlrl}
\mathcal{C}^{0}=\llbracket \Gamma^{000} \rrbracket\{4\} \quad \check{\mathcal{C}}^{1}=\llbracket \Gamma^{001} \rrbracket\{5\} & \check{\mathcal{C}}^{2}=\llbracket \Gamma^{011} \rrbracket\{6\} & \mathcal{C}^{3}=\llbracket \Gamma^{111} \rrbracket\{8\} \\
\widehat{\mathcal{C}}^{1}=\llbracket \Gamma^{100} \rrbracket\{6\} \oplus \llbracket \Gamma_{1}^{010} \rrbracket\{5\} & \widehat{\mathcal{C}}^{2}=\llbracket \Gamma^{110} \rrbracket\{7\} \oplus \llbracket \Gamma_{1}^{101} \rrbracket\{7\} .
\end{array}
$$

Then $\llbracket \Gamma \rrbracket=\operatorname{Cone}\left(\Delta^{0}, \Delta^{1}, \Delta^{2}\right)$ where

$$
\begin{gathered}
\mathcal{C}^{0} \stackrel{\Delta^{0}}{\longrightarrow} \widehat{\mathcal{C}}^{1} \oplus \check{\mathcal{C}}^{1} \stackrel{\Delta^{1}}{\longrightarrow} \widehat{\mathcal{C}}^{2} \oplus \breve{\mathcal{C}}^{2} \stackrel{\Delta^{2}}{\longrightarrow} \mathcal{C}^{3} \\
\Delta^{0}=\left(\begin{array}{l}
d^{\bullet 00} \\
d^{0 \bullet 0} \\
d^{00 \bullet}
\end{array}\right)=\left(\begin{array}{c}
\hat{\Delta}^{0} \\
\hdashline d^{00 \bullet}
\end{array}\right) \quad \Delta^{1}=\left(\begin{array}{cc:c}
d^{1 \bullet 0} & d^{\bullet 10} & 0 \\
d^{10 \bullet} & 0 & d^{\bullet 01} \\
0 & d^{01 \bullet} & d^{0 \bullet 1}
\end{array}\right)=\left(\begin{array}{c:c}
\widehat{\Delta}^{1} & U \\
\hdashline L & d^{0 \bullet 1}
\end{array}\right) \\
\Delta^{2}=\left(d^{11 \bullet} d^{1 \bullet 1} d^{\bullet 11}\right)=\left(\widehat{\Delta}^{2}: d^{\bullet 11}\right) .
\end{gathered}
$$

and

Consider the creation/destruction morphisms of complexes (see Figure 23):

$\llbracket \Gamma^{100} \rrbracket\{6\} \stackrel{\tau}{\rightarrow} \llbracket \Gamma^{000} \rrbracket\{4\} \llbracket \Gamma^{110} \rrbracket\{7\} \stackrel{\eta_{2}}{\rightarrow} \llbracket \Gamma^{010} \rrbracket\{5\} \llbracket \Gamma^{101} \rrbracket\{8\} \stackrel{\eta_{2}}{\rightarrow} \llbracket \Gamma^{011} \rrbracket\{5\}$

$\llbracket \Gamma^{010} \rrbracket\{5\} \stackrel{\sigma}{\rightarrow} \llbracket \Gamma^{000} \rrbracket\{4\} \llbracket \Gamma^{101} \rrbracket\{7\} \stackrel{\eta_{1}}{\rightarrow} \llbracket \Gamma^{100} \rrbracket\{5\} \quad \llbracket \widetilde{\Gamma} \rrbracket\{6\} \stackrel{\eta_{1}}{\rightarrow} \llbracket \Gamma^{001} \rrbracket\{5\}$

$$
\llbracket \Gamma^{101} \rrbracket\{7\} \stackrel{\eta_{2}}{\rightarrow} \llbracket \Gamma^{010} \rrbracket\{5\} \llbracket \Gamma^{001} \rrbracket\{5\} \stackrel{\bar{\varepsilon}_{1}}{\rightarrow} \llbracket \widetilde{\Gamma} \rrbracket\{6\}
$$

Let's define $\sigma^{\prime}=\left(\mathrm{id}+\tau d^{\bullet 00}\right) \sigma: \llbracket \Gamma^{010} \rrbracket\{5\} \rightarrow \llbracket \Gamma^{000} \rrbracket\{4\}$ and

$$
\widehat{H}^{1}=\left(\begin{array}{cc}
0 & \eta_{1} \\
\eta_{2} & \eta_{2}
\end{array}\right) \quad \hat{H}^{0}=\left(\begin{array}{ll}
\tau & \sigma^{\prime}
\end{array}\right) .
$$

Then we have a short exact sequence

$$
0 \longrightarrow \widehat{\mathcal{C}}^{2} \stackrel{\hat{H}^{1}}{\longrightarrow} \hat{\mathcal{C}}^{1} \stackrel{\hat{H}^{0}}{\longrightarrow} \mathcal{C}^{0} \longrightarrow 0
$$

such that $\widehat{\Delta}^{1}$ and $\widehat{\Delta}^{0}$ are splitting morphisms of $\widehat{H}^{1}$ and $\widehat{H}^{0}$ :

$$
\widehat{\Delta}^{1} \widehat{H}^{1}=\mathrm{id}, \quad \hat{H}^{0} \widehat{\Delta}^{0}=\mathrm{id}, \quad \widehat{\Delta}^{0} \hat{H}^{0}+\widehat{H}^{1} \widehat{\Delta}^{1}=\mathrm{id}+\widehat{H}^{1} \widehat{\Delta}^{1} \widehat{\Delta}^{0} \widehat{H}^{0} .
$$




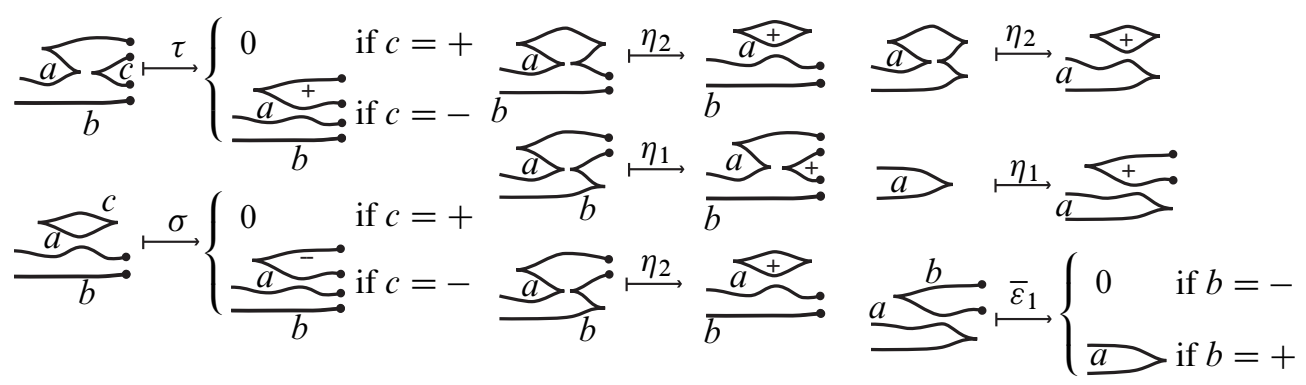
$a, b, c \in\{-,+\}$

Figure 23

Moreover, for the compositions

$$
\llbracket \tilde{\Gamma} \rrbracket\{6\} \stackrel{\eta_{1}}{\longrightarrow} \breve{\mathcal{C}}^{1} \stackrel{d^{0 \bullet 1}}{\longrightarrow} \breve{\mathcal{C}}^{2} \quad \text { and } \quad \check{\mathcal{C}}^{1} \stackrel{U}{\longrightarrow} \widehat{\mathcal{C}}^{2} \stackrel{\widehat{H}^{1}}{\longrightarrow} \widehat{\mathcal{C}}^{1} \stackrel{L}{\longrightarrow} \check{\mathcal{C}}^{2}
$$

we have

$$
d^{0 \bullet 1} \eta_{1}=0 \quad L \hat{H}^{1} U=d^{01 \bullet} \eta_{2} d^{\bullet 01}=0 .
$$

Let's define homotopies $H^{0}: \widehat{\mathcal{C}}^{1} \oplus \breve{\mathcal{C}}^{1} \rightarrow \mathcal{C}^{0}, H^{1}: \widehat{\mathcal{C}}^{2} \oplus \breve{\mathcal{C}}^{2} \rightarrow \widehat{\mathcal{C}}^{1} \oplus \breve{\mathcal{C}}^{1}$ and $H^{2}: \mathcal{C}^{3} \rightarrow$ $\widehat{\mathcal{C}}^{2} \oplus \breve{\mathcal{C}}^{2}$ by

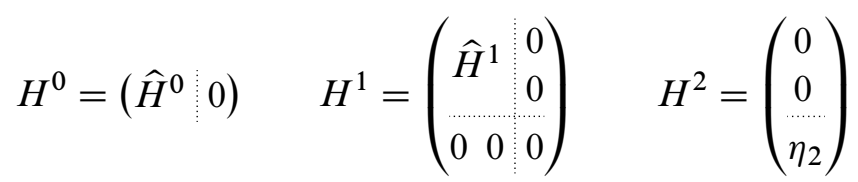

and consider the injection $J$ and retraction $R$

$$
\begin{gathered}
\llbracket \tilde{\Gamma} \rrbracket\{6\} \\
R \|_{J} \\
\mathcal{C}^{0} \underset{H^{0}}{\stackrel{\Delta^{0}}{R}} \widehat{\mathcal{C}}^{1} \oplus \check{\mathcal{C}}^{1} \underset{H^{1}}{\stackrel{\Delta^{1}}{\longrightarrow}} \widehat{\mathcal{C}}^{2} \oplus \breve{\mathcal{C}}^{2} \underset{H^{2}}{\stackrel{\Delta^{2}}{\longrightarrow}} \mathcal{C}^{3}
\end{gathered}
$$

where

$$
\begin{aligned}
& R=\left(\begin{array}{lll}
0 & 0 & \bar{\varepsilon}_{1}
\end{array}\right)\left(\mathrm{id}+\Delta^{0} H^{0}\right)=\left(\bar{\varepsilon}_{1} d^{00 \bullet} \tau \bar{\varepsilon}_{1} d^{00 \bullet} \sigma^{\prime} \bar{\varepsilon}_{1}\right) \\
& J=\left(\mathrm{id}+H^{1} \Delta^{1}\right)\left(\begin{array}{c}
0 \\
0 \\
\eta_{1}
\end{array}\right)=\left(\begin{array}{c}
\eta_{1} d^{\bullet 01} \eta_{1} \\
\eta_{2} d^{\bullet 01} \eta_{1} \\
\eta_{1}
\end{array}\right) .
\end{aligned}
$$

(see Figure 24 for the retraction $R$ ). 


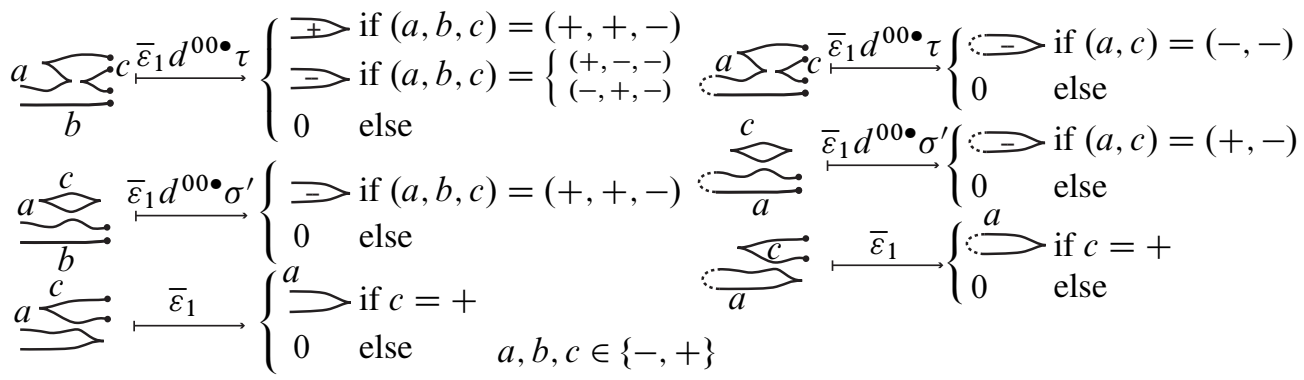

Figure 24: The retraction $R$

From (4-7) and (4-8), we easily verify the relations:

$$
R \Delta^{0}=0, \quad \Delta^{1} J=0, \quad H^{0} J=0, \quad R H^{1}=0, \quad H^{0} H^{1}=0, \quad H^{1} H^{2}=0
$$

$$
R J=\mathrm{id}, J R=\mathrm{id}+\Delta^{0} H^{0}+H^{1} \Delta^{1}, \mathrm{id}+\Delta^{1} H^{1}+H^{2} \Delta^{2}=0 \text {, id }+\Delta^{2} H^{2}=0 \text {. }
$$

Then $\llbracket \tilde{\Gamma} \rrbracket\{6\}[1]$ is a strong deformation retract of $\llbracket \Gamma \rrbracket=\operatorname{Cone}\left(\Delta^{0}, \Delta^{1}, \Delta^{2}\right)$ from Proposition 3.12.

Proof of Proposition 4.12 Let $\Gamma_{1}^{s}$ (resp. $\Gamma_{2}^{s}$ ), $s \in\{0,1\}$ be the cuspidal divides obtained by performing $\Theta_{s}$ splittings at the left hand + double point of $\Gamma_{1}$ (resp. $\Gamma_{2}$ ) in Figure 20. Let's also denote, according to Lemma 4.13, the cuspidal divides $\widetilde{\Gamma}_{1}^{1}$ (resp. $\left.\tilde{\Gamma}_{2}^{1}\right)$ obtained by "retracting" $\Gamma_{1}^{1}\left(\right.$ resp. $\left.\Gamma_{2}^{1}\right)$ (see Figure 25 ). Notice that

$$
\begin{aligned}
& \Gamma_{1}^{0}=\cong \quad \Gamma_{1}^{1}=\geqq \quad \Gamma_{1}^{1}=\geqq \\
& \Gamma_{2}^{0}=\gtreqless \quad \Gamma_{2}^{1}=\gtreqless \quad \tilde{\Gamma}_{2}^{1}=\gtreqless
\end{aligned}
$$

Figure 25

$\llbracket \Gamma_{1}^{0} \rrbracket=\llbracket \Gamma_{2}^{0} \rrbracket$ and $\llbracket \tilde{\Gamma}_{1}^{1} \rrbracket=\llbracket \tilde{\Gamma}_{2}^{1} \rrbracket$. From Lemma 3.14, the differential $d_{1}$ on $\llbracket \Gamma_{1} \rrbracket$ (resp. $d_{2}$ on $\left.\llbracket \Gamma_{2} \rrbracket\right)$ gives us the following cone:

$$
\llbracket \Gamma_{1} \rrbracket=\text { Cone }\left(\llbracket \Gamma_{1}^{0} \rrbracket\{2\} \stackrel{d_{1}^{\bullet}}{\rightarrow} \llbracket \Gamma_{1}^{1} \rrbracket\{4\}\right) \quad\left(\text { resp. } \llbracket \Gamma_{2} \rrbracket=\text { Cone }\left(\llbracket \Gamma_{2}^{0} \rrbracket\{2\} \stackrel{d_{2}^{\bullet}}{\rightarrow} \llbracket \Gamma_{2}^{1} \rrbracket\{4\}\right)\right) .
$$

From Lemma 4.13, there exist strong deformation retractions

$$
\llbracket \Gamma_{1}^{1} \rrbracket \underset{J_{1}}{\stackrel{R_{1}}{\rightleftarrows}} \llbracket \tilde{\Gamma}_{1}^{1} \rrbracket\{6\}[1] \quad \llbracket \Gamma_{2}^{1} \rrbracket \underset{J_{2}}{\stackrel{R_{2}}{\rightleftarrows}} \llbracket \tilde{\Gamma}_{2}^{1} \rrbracket\{6\}[1] .
$$


So from Proposition 3.10, Cone $\left(R_{1} d_{1}^{\bullet}\right)$ (resp. $\left.\operatorname{Cone}\left(R_{2} d_{2}^{\bullet}\right)\right)$ is a strong deformation retract of $\llbracket \Gamma_{1} \rrbracket\left(\right.$ resp. $\left.\llbracket \Gamma_{2} \rrbracket\right)$. Hence it suffices to show that $R_{1} d_{1}^{\bullet}=R_{2} d_{2}^{\bullet}$. Let's consider the splitting diagram of $\llbracket \Gamma_{1}^{0} \rrbracket=\llbracket \Gamma_{2}^{0} \rrbracket$ as $\operatorname{Cone}\left(D^{0}, D^{1}, D^{2}\right)$ (see Figure 26).

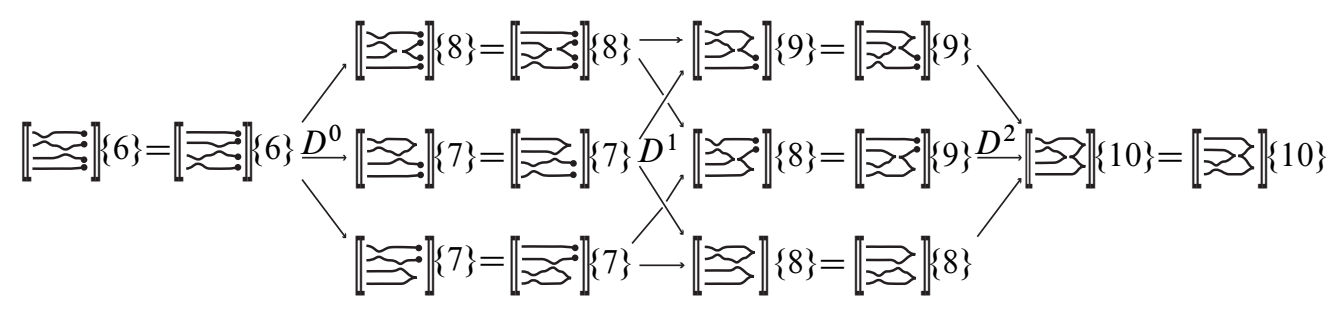

Figure 26

Now $R_{1} d_{1}^{\bullet}$ and $R_{2} d_{2}^{\bullet}$ corresponds to the diagram of Figure 27.

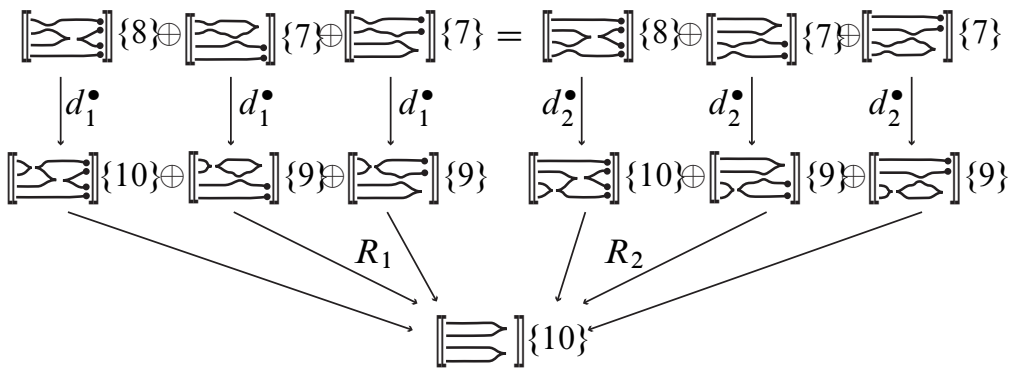

Figure 27

By combining Figure 24 with Figure 6 we easily verify that $R_{1} d_{1}^{\bullet}=R_{2} d_{2}^{\bullet}$.

\subsection{Invariance under type $V$ moves}

Let $\Gamma_{0}$ and $\Gamma_{+}$(resp. $\Gamma_{-}$) be OMS-divides which differ only by a type $\mathrm{V}^{*}+$ (resp. type $\mathrm{V}^{*}-$ ) move (see Figure 28 ).

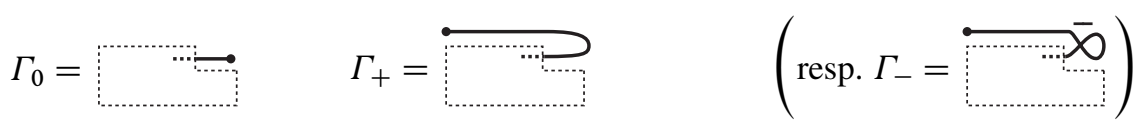

Figure 28: Type V moves 
Proposition 4.14 The complexes $\llbracket \Gamma_{0} \rrbracket, \llbracket \Gamma_{+} \rrbracket$ and $\llbracket \Gamma_{-} \rrbracket$ have the same homology.

Proof From Lemma 3.14, we can see $\llbracket \Gamma_{+} \rrbracket$ as the cone of the surjective morphism as in Figure 29.

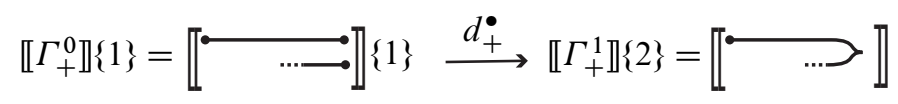

Figure 29: Splitting diagram for type $V_{+}$move

Let's consider the creation/destruction morphisms $\eta_{1}: \llbracket \Gamma_{+}^{1} \rrbracket\{2\} \rightarrow \llbracket \Gamma_{+}^{0} \rrbracket\{1\}$ (right inverse of $\left.d_{+}^{\bullet}\right), \bar{\eta}_{1}: \llbracket \Gamma \rrbracket \rightarrow \llbracket \Gamma_{+}^{0} \rrbracket\{1\}$ and $\varepsilon_{1}: \llbracket \Gamma_{+}^{0} \rrbracket\{1\} \rightarrow \llbracket \Gamma \rrbracket$ defined in Figure 30 .

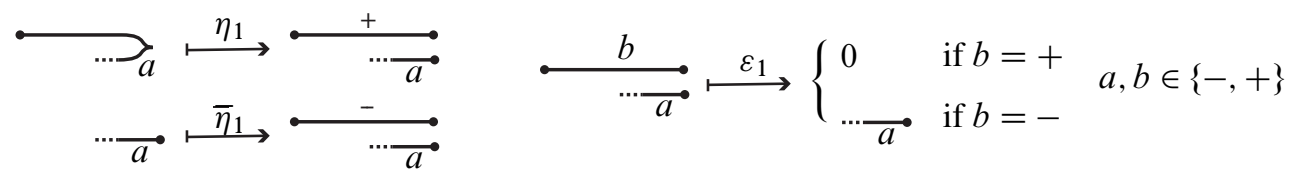

Figure 30

Let $j=\left(\mathrm{id}+\eta_{1} d^{\bullet}\right) \bar{\eta}_{1}$ and $r=\varepsilon_{1}$. Then from the following diagram

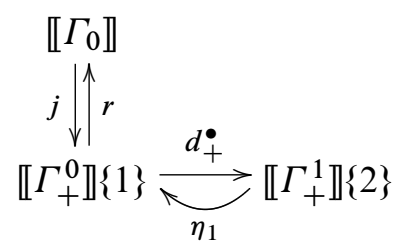

where

$$
\begin{aligned}
d_{+}^{\bullet} j & =0, & r j & =\mathrm{id}, \\
j r & =\mathrm{id}+\eta_{1} d_{+}^{\bullet}, & d_{+}^{\bullet} \eta_{1} & =\mathrm{id}
\end{aligned}
$$

we deduce using Proposition 3.12 that $\llbracket \Gamma_{0} \rrbracket$ is a strong deformation retract of $\llbracket \Gamma_{+} \rrbracket=$ Cone $\left(d_{+}^{\bullet}\right)$. So they have the same homology.

On the other hand, from Lemma 3.14, $\llbracket \Gamma_{-} \rrbracket=\operatorname{Cone}\left(\Delta^{0}, \Delta^{1}\right)[-1]$ :

$\llbracket \Gamma_{-}^{10} \rrbracket\{-3\} \stackrel{\Delta^{0}}{\rightarrow} \llbracket \Gamma_{-}^{00} \rrbracket\{-1\} \oplus \llbracket \Gamma_{-}^{11} \rrbracket\{-2\} \stackrel{\Delta^{1}}{\rightarrow} \llbracket \Gamma_{-}^{01} \rrbracket\{3\} \quad \Delta^{0}=\left(\begin{array}{l}d_{-}^{\bullet 0} \\ d_{-}^{1} \bullet\end{array}\right) \quad \Delta^{1}=\left(d_{-}^{0 \bullet} d_{-}^{\bullet 1}\right)$ 


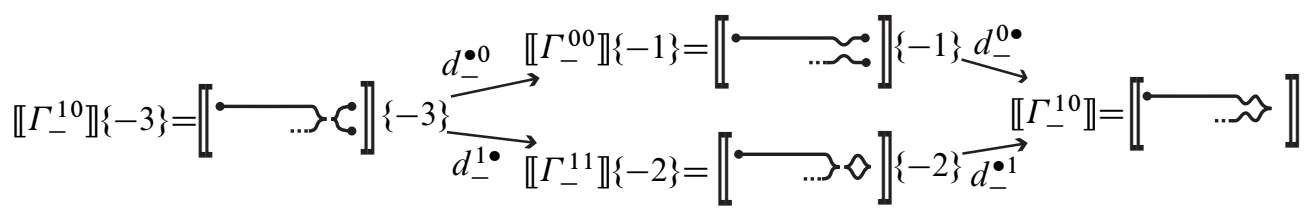

Figure 31: Splitting diagram for type $V_{-}$move

Let's consider the morphisms

$$
\begin{array}{rr}
\llbracket \Gamma_{-}^{00} \rrbracket\{-1\} \stackrel{\tau}{\rightarrow} \llbracket \Gamma_{-}^{10} \rrbracket\{-3\}, & \llbracket \Gamma_{-}^{11} \rrbracket\{-2\} \stackrel{\sigma}{\rightarrow} \llbracket \Gamma_{-}^{10} \rrbracket\{-3\}, \\
\llbracket \Gamma_{-}^{01} \rrbracket \stackrel{\eta_{1}}{\rightarrow} \llbracket \Gamma_{-}^{00} \rrbracket\{-1\}, & \llbracket \Gamma_{-}^{01} \rrbracket \stackrel{\eta_{2}}{\rightarrow} \llbracket \Gamma_{-}^{11} \rrbracket\{-2\}
\end{array}
$$

defined in Figure 32.

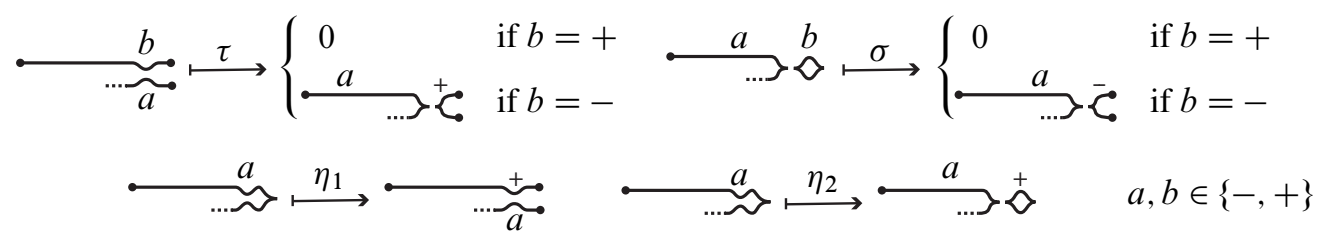

Figure 32

Then from the diagram

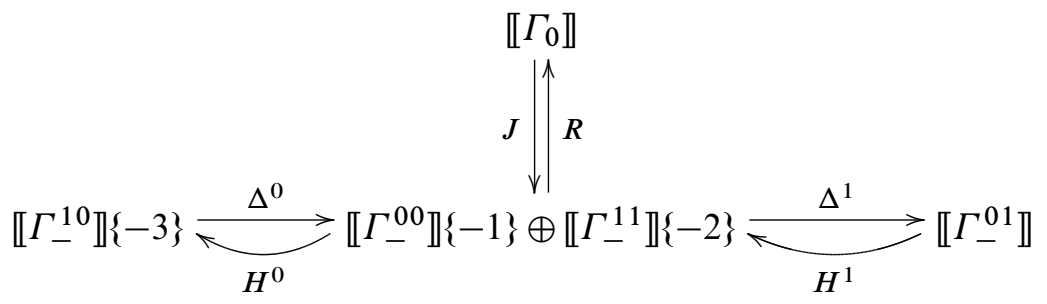

where $\left.J=\left(\begin{array}{l}\eta_{1} \\ \eta_{2}\end{array}\right), \quad R=\left(\begin{array}{ll}0 & \bar{\varepsilon}_{2}\end{array}\right), \quad H^{0}=\left(\begin{array}{l}\tau\left(\mathrm{id}+\tau d^{\bullet 0}\right.\end{array}\right) \sigma\right) \quad$ and $\quad H^{1}=\left(\begin{array}{l}\eta_{1} \\ \eta_{2}\end{array}\right)$

we deduce that $\llbracket \Gamma_{0} \rrbracket$ is a strong deformation retract of $\llbracket \Gamma_{-} \rrbracket=\operatorname{Cone}\left(\Delta^{0}, \Delta^{1}\right)[-1]$. They have the same homology. 


\subsection{Invariance under type VI moves}

Let $\Gamma$ and $\Gamma_{+}$(resp. $\Gamma_{-}$) be OMS-divides which differ only by a type $\mathrm{VI}_{+}$(resp. type $\mathrm{VI}_{-}$) move (see Figure 33).

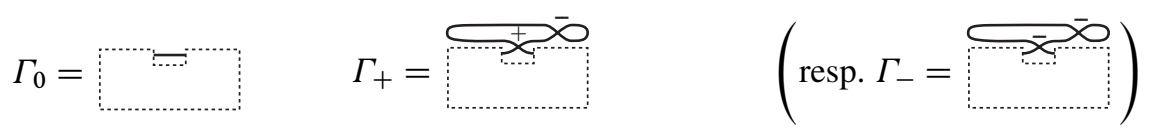

Figure 33: Type VI moves

Proposition 4.15 The complexes $\llbracket \Gamma_{0} \rrbracket, \llbracket \Gamma_{+} \rrbracket$ and $\llbracket \Gamma_{-} \rrbracket$ have the same homology.

We will break down the proof in two steps: the result is an immediate consequence of the following two lemmas.

Lemma 4.16 Let $\Gamma$ and $\tilde{\Gamma}$ be cuspidal divides as depicted in Figure 34.

$$
\Gamma=\complement_{\ldots} \ldots \bar{\Gamma} \quad \tilde{\Gamma}=\lessdot \ldots
$$

Figure 34

Then $\llbracket \tilde{\Gamma} \rrbracket$ is a strong deformation retract of $\llbracket \Gamma \rrbracket$.

Proof Let's apply Lemma 3.14 to $\llbracket \Gamma \rrbracket$. We have a splitting diagram as depicted in Figure 35.

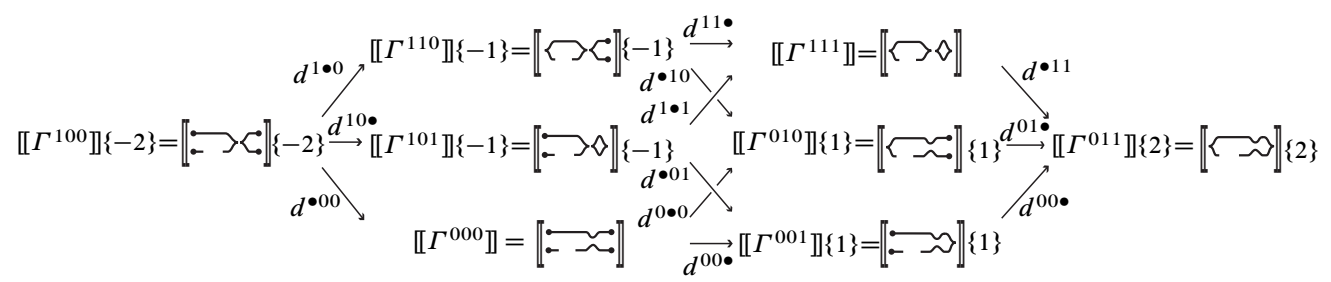

Figure 35: Splitting diagram of $\llbracket \Gamma \rrbracket$

Let's denote:

$$
\begin{gathered}
\mathcal{C}^{0}=\llbracket \Gamma^{100} \rrbracket\{-2\} \quad \mathcal{C}^{1}=\llbracket \Gamma^{110} \rrbracket\{-1\} \oplus\left(\llbracket \Gamma^{101} \rrbracket\{-1\} \oplus \llbracket \Gamma^{000} \rrbracket\right) \\
\mathcal{C}^{2}=\llbracket \Gamma^{111} \rrbracket \oplus\left(\llbracket \Gamma^{010} \rrbracket\{1\} \oplus \llbracket \Gamma^{001} \rrbracket\{1\}\right) \quad \mathcal{C}^{3}=\llbracket \Gamma^{011} \rrbracket\{2\} \\
\Delta^{0}=\left(\begin{array}{c}
d^{1 \bullet 0} \\
d^{10 \bullet} \\
d^{\bullet 00}
\end{array}\right) \quad \Delta^{1}=\left(\begin{array}{ccc}
d^{11} & d^{1 \bullet 1} & 0 \\
d^{\bullet 10} & 0 & d^{0 \bullet 0} \\
0 & d^{\bullet 01} & d^{00 \bullet}
\end{array}\right) \quad \Delta^{2}=\left(d^{\bullet 11} d^{01 \bullet} d^{0 \bullet 1}\right) .
\end{gathered}
$$


Then $\llbracket \Gamma \rrbracket=\operatorname{Cone}\left(\Delta^{0}, \Delta^{1}, \Delta^{2}\right)[-1]$ where

$$
\mathcal{C}^{0} \stackrel{\Delta^{0}}{\longrightarrow} \mathcal{C}^{1} \stackrel{\Delta^{1}}{\longrightarrow} \mathcal{C}^{2} \stackrel{\Delta^{2}}{\longrightarrow} \mathcal{C}^{3}
$$

Let's consider the creation / destruction morphisms

$$
\begin{array}{ccc}
\llbracket \Gamma^{101} \rrbracket\{-1\} \stackrel{\sigma}{\longrightarrow} \llbracket \Gamma^{100} \rrbracket\{-2\} & \llbracket \Gamma^{111} \rrbracket \stackrel{\sigma}{\longrightarrow} \llbracket \Gamma^{110} \rrbracket\{-1\} & \llbracket \Gamma^{011} \rrbracket\{2\} \stackrel{\eta_{2}}{\longrightarrow} \llbracket \Gamma^{111} \rrbracket \\
\llbracket \Gamma^{000} \rrbracket \stackrel{\tau}{\longrightarrow} \llbracket \Gamma^{100} \rrbracket\{-2\} & \llbracket \Gamma^{010} \rrbracket\{1\} \stackrel{\eta_{1}}{\longrightarrow} \llbracket \Gamma^{000} \rrbracket & \llbracket \tilde{\Gamma} \rrbracket \stackrel{\eta_{1}}{\longrightarrow} \llbracket \Gamma^{110} \rrbracket\{-1\} \\
& \llbracket \Gamma^{001} \rrbracket\{1\} \stackrel{\eta_{2}}{\longrightarrow} \llbracket \Gamma^{101} \rrbracket\{-1\} & \llbracket \Gamma^{110} \rrbracket\{-1\} \stackrel{\bar{\varepsilon}_{1}}{\longrightarrow} \llbracket \tilde{\Gamma} \rrbracket
\end{array}
$$

as depicted in Figure 36.

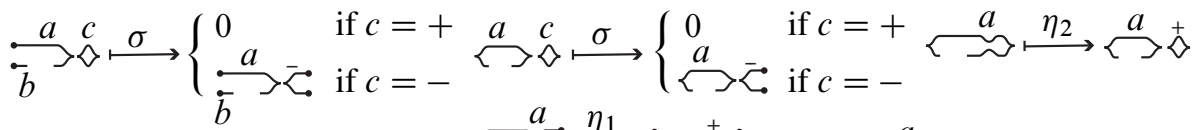

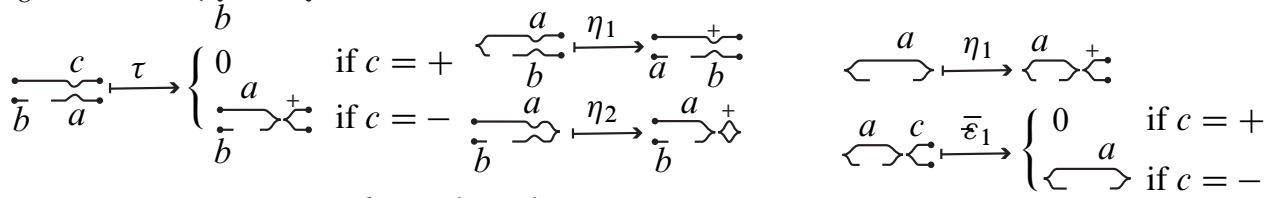

$$
\begin{aligned}
& a, b, c \in\{-,+\}
\end{aligned}
$$

Figure 36

We define homotopies $H^{0}: \mathcal{C}^{1} \rightarrow \mathcal{C}^{0}, H^{1}: \mathcal{C}^{2} \rightarrow \mathcal{C}^{1}$ and $H^{2}: \mathcal{C}^{3} \rightarrow \mathcal{C}^{2}$ :

$$
H^{0}=\left(\begin{array}{lll}
0 & \left(1+\tau d^{\bullet 00}\right) \sigma & \tau
\end{array}\right), H^{1}=\left(\begin{array}{ccc}
\sigma & 0 & 0 \\
\eta_{2} d^{00 \bullet} \eta_{1} d^{\bullet 10} \sigma & \eta_{2} d^{00 \bullet} \eta_{1} & \eta_{2} \\
\eta_{1} d^{\bullet 10} \sigma & \eta_{1} & 0
\end{array}\right), H^{2}=\left(\begin{array}{c}
\eta_{2} \\
0 \\
0
\end{array}\right)
$$

together with retraction and inclusion maps $R: \mathcal{C}^{1} \rightarrow \llbracket \tilde{\Gamma} \rrbracket$ and $J: \llbracket \tilde{\Gamma} \rrbracket \rightarrow \mathcal{C}^{1}$ :

$$
\begin{gathered}
R=\left(\begin{array}{c:cc}
\bar{\varepsilon}_{1} & 0 & 0
\end{array}\right)\left(\mathrm{id}+\Delta^{0} H^{0}\right)=\left(\bar{\varepsilon}_{1} \bar{\varepsilon}_{1} d^{1 \bullet 0} \tau d^{\bullet 00} \sigma \bar{\varepsilon}_{1} d^{1 \bullet 0} \tau\right) \\
J=\left(\mathrm{id}+H^{1} \Delta^{1}\right)\left(\begin{array}{c}
\eta_{1} \\
0 \\
0
\end{array}\right)=\left(\begin{array}{c}
\eta_{1} \\
\eta_{2} d^{00 \bullet} \eta_{1} d^{\bullet 10} \eta_{1} \\
\eta_{1} d^{\bullet 10} \eta_{1}
\end{array}\right)
\end{gathered}
$$


such that we have the diagram:

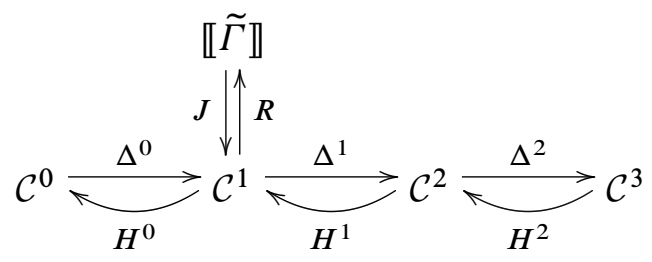

We easily verify that

$$
R \Delta^{0}=0, \quad \Delta^{1} J=0, \quad H^{0} J=0, \quad R H^{1}=0, \quad H^{0} H^{1}=0, \quad H^{1} H^{2}=0,
$$

$R J=\mathrm{id}, J R=\mathrm{id}+\Delta^{0} H^{0}+H^{1} \Delta^{1}, \mathrm{id}+\Delta^{1} H^{1}+H^{2} \Delta^{2}=0, \mathrm{id}+\Delta^{2} H^{2}=0$.

Then from Proposition 3.12, $\llbracket \tilde{\Gamma} \rrbracket$ is a strong deformation retract of $\llbracket \Gamma \rrbracket$.

Lemma 4.17 We have strong deformation retractions $r_{+}$(resp. $r_{-}$) with injection $j_{+}$ (resp. $j_{-}$) pictured in Figure 37.

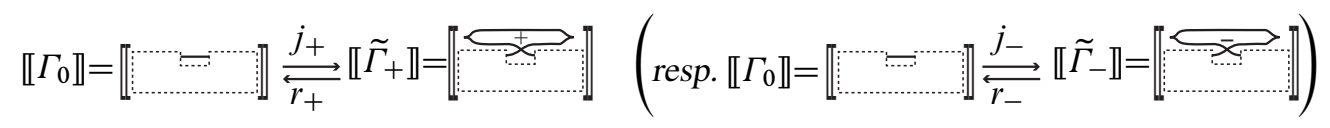

Figure 37

Proof From Lemma 3.14, $\llbracket \widetilde{\Gamma}_{+} \rrbracket=\operatorname{Cone}\left(d_{+}\right)$and $\llbracket \widetilde{\Gamma}_{-} \rrbracket=\operatorname{Cone}\left(d_{-}\right)[-1]$ where $d_{+}$ and $d_{-}$are shown in Figure 38.

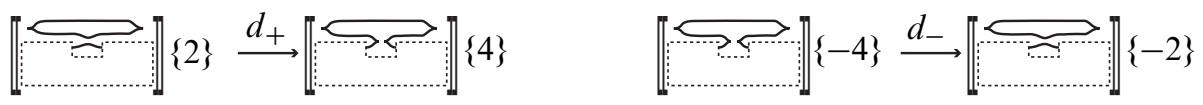

Figure 38

For $\llbracket \Gamma_{+} \rrbracket$, using Proposition 3.12, we deduce the strong deformation retraction from the following diagram of Figure 39 since $d_{+} \eta_{2}=\mathrm{id}, r_{+} \eta_{2}=0, d_{+} j_{+}=0, r_{+} j_{+}=\mathrm{id}$ and $j_{+} r_{+}=\mathrm{id}+\eta_{2} d_{+}$.

Similarly for $\llbracket \Gamma_{-} \rrbracket$ we deduce the strong deformation retraction from the diagram of Figure 40 since $\varepsilon_{2} d_{-}=\mathrm{id}, r_{-} d_{-}=0, \varepsilon_{2} j_{-}=0, r_{-} j_{-}=\mathrm{id}$ and $j_{-} r_{-}=\mathrm{id}+d_{-} \varepsilon_{2}$. 

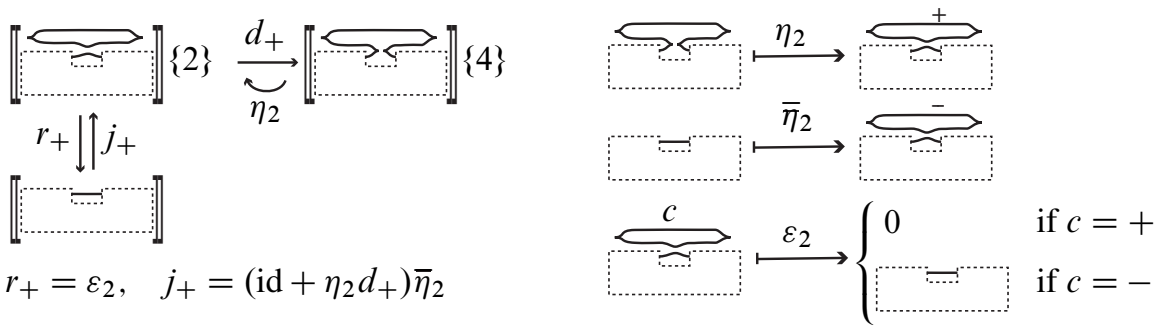

$r_{+}=\varepsilon_{2}, \quad j_{+}=\left(\mathrm{id}+\eta_{2} d_{+}\right) \bar{\eta}_{2}$

Figure 39
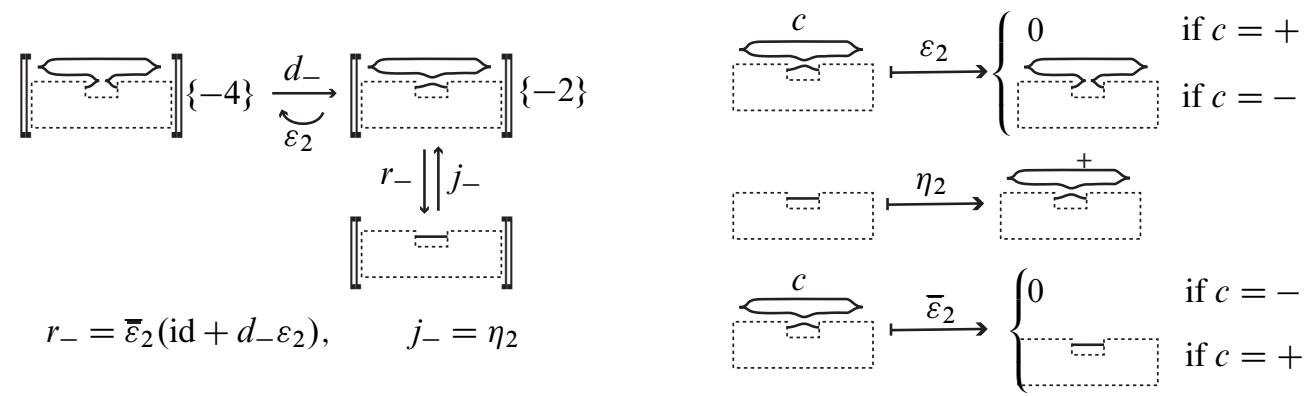

Figure 40

\section{References}

[1] N A'Campo, Real deformations and complex topology of plane curve singularities, Ann. Fac. Sci. Toulouse Math. (6) 8 (1999) 5-23 MR1721511

[2] O Couture, Strongly invertible links and divides, Topology 47 (2008) 316-350 MR2422530

[3] O Couture, B Perron, Representative braids for links associated to plane immersed curves, J. Knot Theory Ramifications 9 (2000) 1-30 MR1749500

[4] J W Morgan, H Bass (editors), The Smith conjecture, Pure and Applied Math. 112, Academic Press, Orlando, FL (1984) MR758459 Papers presented at the symposium held at Columbia University, New York, 1979

[5] O Viro, Remarks on the definition of Khovanov homology arXiv:math.GT/0202199

Institut de Mathématiques de Bourgogne, Université de Bourgogne

UFR Sciences et Techniques, 9 avenue Alain Savary, BP 47870, 21078 Dijon Cedex, France ocouture@u-bourgogne.fr

http://math.u-bourgogne.fr/IMB/

Received: 17 February $2009 \quad$ Revised: 27 August 2009 